\title{
ERROR AND STABILITY ESTIMATES FOR SURFACE-DIVERGENCE FREE RBF INTERPOLANTS ON THE SPHERE
}

\author{
EDWARD J. FUSELIER, FRANCIS J. NARCOWICH, JOSEPH D. WARD, \\ AND GRADY B. WRIGHT
}

\begin{abstract}
Recently, a new class of surface-divergence free radial basis function interpolants has been developed for surfaces in $\mathbb{R}^{3}$. In this paper, several approximation results for this class of interpolants will be derived in the case of the sphere, $\mathbb{S}^{2}$. In particular, Sobolev-type error estimates are obtained, as well as optimal stability estimates for the associated interpolation matrices. In addition, a Bernstein estimate and an inverse theorem are also derived. Numerical validation of the theoretical results is also given.
\end{abstract}

\section{INTRODUCTION}

In [35, a new tool was developed, based on radial basis functions (RBFs), for fitting a divergence-free vector field tangent to a two-dimensional orientable surface $\mathcal{P} \subset R^{3}$ to samples of such a field taken at scattered sites on $\mathcal{P}$. The central idea in [35] was to construct positive definite kernels, "surface-divergence free RBFs", to obtain a surface-divergence free vector field to fit a given finite set of tangent vectors on the surface $\mathcal{P}$.

An important application for these new surface-divergence free kernel methods is modeling the velocity field of an incompressible fluid whose flow is constrained to the surface $\mathcal{P}$. In this case, the incompressibility assumption gives rise to the constraint that the velocity field has vanishing surface-divergence. This type of problem arises in atmospheric sciences and oceanography in which case $\mathcal{P}$ is the entire surface of the sphere $\left(\mathbb{S}^{2}\right)$ or some portion of it. For example, the shallow water wave equations describe the nonlinear flow of an incompressible fluid in a single hydrostatic layer and are used not only as a simplified model for the horizontal dynamics of the atmosphere [43, but also as a model for tidal motion [22]. The incompressibility constraint also arises in the barotropic vorticity equations, which

Received by the editor February 8, 2008 and, in revised form, August 25, 2008.

2000 Mathematics Subject Classification. Primary 41A05, 41A63; Secondary 76M25, 86-08, $86 \mathrm{~A} 10$.

Key words and phrases. Sphere, vector fields, incompressible fluids, radial basis functions, numerical modeling, stream function.

The second author's research was supported by grant DMS-0504353 from the National Science Foundation.

The third author's research was supported by grant DMS-0504353 from the National Science Foundation.

The fourth author's research was supported by grant ATM-0801309 from the National Science Foundation.

(C)2009 American Mathematical Society Reverts to public domain 28 years from publication 
are used to model the 500-mb short-term weather forecasts in mid-latitudes [14, pp. 108-110]. These new surface-divergence free kernel methods could be used either to interpolate velocity fields generated from simulations of these models, or directly used in the simulation as the representation of the velocity fields.

Divergence-free RBFs and curl-free RBFs were introduced several years ago for modeling velocity fields and the magnetic fields in $\mathbb{R}^{3}$ 29. However, when restricted to a sphere or to a surface $\mathcal{P}$, these RBFs, which are constructed to be divergence free or curl free in $\mathbb{R}^{3}$, lose those properties on $\mathcal{P}$.

The specifics of the new method are as follows. Suppose that $\mathcal{P}$ has a fixed orientation and $x$ and $y$ belong to $\mathcal{P}$. Let $\mathbf{n}_{x}$ and $\mathbf{n}_{y}$ denote the normals to $x$ and $y$, respectively, and for any vector $\mathbf{a}=\left[a_{1}, a_{2}, a_{3}\right]^{T} \in \mathbb{R}^{3}$ define

$$
\mathrm{X}_{\mathbf{a}}=\left[\begin{array}{ccc}
0 & -a_{3} & a_{2} \\
a_{3} & 0 & -a_{1} \\
-a_{2} & a_{1} & 0
\end{array}\right]
$$

Note that for $\mathbf{b} \in \mathbb{R}^{3}, \mathrm{X}_{\mathbf{a}} \mathbf{b}=\mathbf{a} \times \mathbf{b}$. The surface-divergence free $\mathrm{RBF}$ is given by

$$
\Psi(x, y):=\mathbf{X}_{\mathbf{n}_{x}}(\underbrace{-\nabla \nabla^{T} \boldsymbol{\psi}(x-y)}_{\Psi_{\text {curl }}}) \mathbf{X}_{\mathbf{n}_{y}}^{T}=\mathbf{X}_{\mathbf{n}_{x}} \Psi_{c u r l}(x-y) \mathbf{X}_{\mathbf{n}_{y}}^{T},
$$

where $\boldsymbol{\psi}$ is a positive definite or an order 1 positive definite RBF. The kernel $\Psi_{\text {curl }}:=-\nabla \nabla^{T} \boldsymbol{\psi}$ is the negative of the 3D Hessian of $\boldsymbol{\psi}$ and is a $3 \times 3$ matrixvalued RBF whose columns are curl free [29, 8]. The kernel $\Psi(x, y)$ takes vectors tangent to $\mathcal{P}$ at $y$ and outputs vectors tangent at $x$. The output vector field is surface-divergence free [35, Theorem 1].

Let $X=\left\{x_{1}, \ldots, x_{N}\right\} \subset \mathcal{P}$ and suppose $\left\{\mathbf{t}_{1}, \ldots, \mathbf{t}_{N}\right\}$ are the corresponding samples of some vector field tangent to $\mathcal{P}$ at these points. Then the surface-divergence free $\mathrm{RBF}$ interpolant to this data is given by

$$
\mathbf{t}(x)=\sum_{k=1}^{N} \Psi\left(x, x_{k}\right) \mathbf{s}_{k},
$$

where $\mathbf{t}\left(x_{j}\right)=\mathbf{t}_{j}$ and $\mathbf{s}_{j}$ is tangent to $\mathcal{P}$ at $x_{j}, j=1, \ldots, N$. These requirements are met by solving the following linear system of equations for the $\mathbf{s}_{k}$ 's:

$$
\mathbf{t}_{j}=\sum_{k=1}^{N} \Psi\left(x_{j}, x_{k}\right) \mathbf{s}_{k}, j=1, \ldots, N .
$$

In Theorem 2 of 35 , it is shown that this linear system is positive definite, which guarantees a unique solution. In the actual implementation of the method, one can reduce the $3 N \times 3 N$ linear system (1.4) to a $2 N \times 2 N$ system by introducing coordinates and bases for the various tangent planes of $\mathcal{P}$ involved. In the specific case of $\mathcal{P}=\mathbb{S}^{2}$, these details are reviewed in section 3.2. for a general orientable surface $\mathcal{P}$ see [35, §3.1].

There is a bonus. In addition to producing a fit of the $\mathbf{t}_{j}$ 's, these new interpolants can also be used to obtain a stream function with level curves having the $\mathbf{t}_{j}$ 's as tangents at the $x_{j}$ 's at virtually no extra cost. This is important since in many applications from oceanography and atmospheric sciences, it is sometimes more desirable to model stream functions instead of velocity fields since they are a better tool for analyzing flows and fluxes on the surface of the sphere (cf. [2, 11]). 
The details are as follows. Suppose the field has been fit, i.e., the coefficients $\mathbf{s}_{k}$ in (1.3) have been determined. Let $\Upsilon(x, y):=\nabla^{T} \boldsymbol{\psi}(x-y) \mathrm{X}_{\mathbf{n}_{y}}^{T}$ and define the scalar valued function

$$
F(x):=\sum_{k=1}^{N} \Upsilon\left(x, x_{k}\right) \mathbf{s}_{k} .
$$

It follows immediately that the level curves of $F(x)$ are tangent to the vector field $\mathbf{t}(x)$ at $x=x_{j}, j=1, \ldots, N$, and consequently $F(x)$ is a stream function for $\mathbf{t}(x)$.

In this paper the basic approximation properties of these kernels will be developed in the case that the surface $\mathcal{P}$ is $\mathbb{S}^{2}$. In particular, Sobolev-type error bounds are obtained when approximating a divergence-free function $\mathbf{f}$ by divergence-free interpolants. In addition, optimal estimates on the smallest eigenvalue of the interpolation matrix are obtained, a Bernstein inequality together with inverse theorem are derived and finally numerical results confirming the theoretical expectations for both approximation rates and stability are presented. Prior work, related to this paper, appeared in [7. However, neither optimal error estimates nor stability results were obtained in [7]. Moreover, the results could not be adapted to surfaces other than the sphere.

This paper consists of five sections. Section 2 reviews spherical harmonics and vector spherical harmonics, Sobolev spaces of vector fields, and develops the native spaces needed to analyze both the stability of interpolation matrices as well as approximation error rates. In section 3 we obtain pointwise error bounds and discuss stability for the interpolation matrices involved. For certain kernels, we show that the stability is optimal. In section 4, we begin by obtaining Sobolev error estimates for interpolation with surface-divergence free vector spherical polynomials. These results are then applied to finding error estimates for functions too rough to be in the native space, for those in the native space, and then for those smoother than required for being in that space; i.e., the "doubling trick" introduced in [38]. Once we have done this, we will turn to Bernstein inequalities and corresponding inverse theorems. Finally, section 5 contains numerical validation of the predicted theoretical estimates for errors and for stability.

\section{FunCtion spaCes on the SPHERE}

Our notation for Sobolev spaces on $\mathbb{R}^{n}$ will follow [1. If $M$ is a smooth manifold and $x \in M$, we denote the space of tangent vectors to $M$ at $x$ by $T_{x} M$, and let $T M$ denote the tangent bundle of $M$. We will focus on the manifold $\mathbb{S}^{2}$. The geodesic distance will be denoted by $d(x, y)$. The $L_{2}$ inner product on the sphere will be denoted by $(\cdot, \cdot)$. Vector fields will be written in boldface to distinguish them from scalar functions. We will often view tangent vector fields to $\mathbb{S}^{2}$ as being embedded in $\mathbb{R}^{3}$. This should cause no confusion. When the context is clear we will use "divergence" to mean "surface-divergence." We denote the surface gradient by $\nabla^{*}$ and the Laplace-Beltrami operator by $\Delta$.

The operator $\mathbf{n} \times \nabla^{*}$, where $\mathbf{n}$ is the unit normal to $\mathbb{S}^{2}$, is the generator of an infinitesimal rotation about $\mathbf{n}$. We will denote it by $\mathbf{L}$. (In quantum mechanics, $\mathbf{L}$ denotes $-i \mathbf{n} \times \nabla^{*}$, which is the angular momentum operator.) There is another important, well-known fact that we will need. 
Proposition 2.1. If $\mathbf{f}$ is a $C^{1}$ tangential vector field on $\mathbb{S}^{2}$ such that $\operatorname{div}_{\mathbb{S}^{2}}(\mathbf{f})=0$, then there is a function $f \in C^{2}\left(\mathbb{S}^{2}\right)$, unique up to an additive constant, such that $\mathbf{f}=\mathbf{L} f$.

Proof. By the Poincaré Lemma and the fact that $\mathbb{S}^{2}$ is simply connected, every smooth, closed 1-form on $\mathbb{S}^{2}$ is exact. Moreover, $\operatorname{div}_{\mathbb{S}^{2}}(\mathbf{f})=0$ is equivalent to $\mathbf{f} \times \mathbf{n} \cdot d \mathbf{x}=0$, i.e., $\mathbf{f} \times \mathbf{n} \cdot d \mathbf{x}$ is closed, and therefore exact. Thus there is a function $f \in C^{2}\left(\mathbb{S}^{2}\right)$ such that $\mathbf{f} \times \mathbf{n}=\nabla^{*} f$, from which we see that $\mathbf{f}=\mathbf{L} f$.

2.1. Scalar and vector spherical harmonics. Spherical harmonics are eigenfunctions of the Laplace-Beltrami operator on the sphere, and they form an orthonormal basis for $L_{2}\left(\mathbb{S}^{n}\right)$ [6, 26]. We let $\left\{Y_{l, m} \mid 1 \leq m \leq d_{l}\right\}$ denote the orthonormal basis of spherical harmonics of degree $l$, which is the eigenspace corresponding to the eigenvalue $\lambda_{l}=l(l+n-1)$. Thus every function in $L_{2}\left(\mathbb{S}^{n}\right)$ has a Fourier representation of the form

$$
f(x)=\sum_{l=0}^{\infty} \sum_{m=1}^{d_{l}} \widehat{f}(l, m) Y_{l, m}(x) \quad \text { with } \quad \widehat{f}(l, m)=\left(f, Y_{l, m}\right) .
$$

From this representation we also have various Sobolev spaces, whose norms are given via

$$
\|f\|_{H^{\tau}\left(\mathbb{S}^{n}\right)}^{2}=\sum_{l=0}^{\infty} \sum_{m=1}^{d_{l}}\left(1+\lambda_{l}\right)^{\tau}|\widehat{f}(l, m)|^{2} .
$$

In the case of $\mathbb{S}^{2}$, we have $d_{l}=2 l+1$ and $\lambda_{l}=l(l+1)$.

Sobolev spaces on the sphere can also be defined in terms of charts. Let $\mathcal{A}=$ $\left\{U_{j}, \psi_{j}\right\}_{j=1}^{N}$ be an atlas of charts for $M$. With such an atlas, one always has an associated partition of unity. That is, a collection $\left\{\chi_{j}: \mathbb{S}^{n} \rightarrow \mathbb{R}\right\}_{j=1}^{N}$ of $C^{\infty}$ functions that satisfy

$$
\chi_{j} \geq 0, \quad \operatorname{supp}\left(\chi_{j}\right) \subseteq U_{j}, \quad \sum_{1}^{N} \chi_{j}=1 \text { on } \mathbb{S}^{n} .
$$

Also, for $f: M \rightarrow \mathbb{R}$ we define the projections $\pi_{j}(f): \mathbb{R}^{n} \rightarrow \mathbb{R}$ by

$$
\pi_{j}\left(\chi_{j} f\right)=\left\{\begin{array}{cl}
\chi_{j} f\left(\psi^{-1}(x)\right) & x \in B(0,1) \\
0 & \text { otherwise }
\end{array}\right.
$$

The Sobolev space $W_{p}^{\tau}\left(\mathbb{S}^{n}\right)$ can be defined by

$$
W_{p}^{\tau}\left(\mathbb{S}^{n}\right):=\left\{f \in L^{2}\left(\mathbb{S}^{n}\right): \pi_{j}\left(\chi_{j} f\right) \in W_{p}^{\tau}\left(\mathbb{R}^{n}\right) \text { for } j=1, \ldots, N\right\} .
$$

The norm for this space is defined by

$$
\|f\|_{W_{p}^{\tau}\left(\mathbb{S}^{n}\right)}=\left(\sum_{j=1}^{N}\left\|\pi_{j}\left(\chi_{j} f\right)\right\|_{W_{p}^{\tau}\left(\mathbb{R}^{n}\right)}^{2}\right)^{1 / 2} .
$$

We should mention that the spaces are independent of the choice of charts, and when different charts are used the norms are equivalent 21]. Further, we have $W_{2}^{\tau}\left(\mathbb{S}^{n}\right)=H^{\tau}\left(\mathbb{S}^{n}\right)$ with equivalent norms [10, Chapter II].

There is a vectorial analogue of Fourier expansions on the sphere, where spherical harmonics are replaced by vector spherical harmonics [6]. These are used in electrodynamics [17, Section 16.2], although they are certainly less familiar than the spherical harmonics. 
The vector spherical harmonics are arranged in three families, only one of which, the surface-divergence free family, is employed here. We define them via the formulas

$$
\mathbf{y}_{l, m}=\mathbf{L} Y_{l, m} / \sqrt{l(l+1)}, l=1,2, \ldots, \quad m=1, \ldots, 2 l+1 .
$$

In addition, we define the spaces

$$
\Sigma_{l}=\operatorname{span}\left\{\mathbf{y}_{l, m} \mid 1 \leq m \leq 2 l+1\right\} \quad \text { and } \quad \Sigma_{L}=\bigoplus_{l=1}^{L} \Sigma_{l} .
$$

The $\mathbf{y}_{l, m}$ 's are orthonormal in the sense that $\int_{\mathbb{S}^{2}} \mathbf{y}_{j, k}^{T}(x) \mathbf{y}_{l, m}(x) d \mu(x)=\delta_{j, l} \delta_{k, m}$. If $\mathbf{f}$ is in the $L_{2}$ closure of the span of these vector spherical harmonics, then its orthogonal series in the $\mathbf{y}_{l, m}$ 's has (scalar) expansion coefficients given by

$$
\widetilde{\mathbf{f}}(l, m):=\int_{\mathbb{S}^{2}} \mathbf{f}^{T}(x) \mathbf{y}_{l, m}(x) d \mu(x) .
$$

Recall that by Proposition 2.1 every $C^{1}$ divergence-free tangent field $\mathbf{f}$ has the form $\mathbf{f}=\mathbf{L} f$. To obtain the $\widetilde{\mathbf{f}}(l, m)$ 's coefficients in terms of the corresponding $\widehat{f}(l, m)$ 's, note that

$$
\begin{aligned}
\tilde{\mathbf{f}}(l, m) & =\int_{\mathbb{S}^{2}}(\mathbf{L} f)^{T} \mathbf{y}_{l, m} d \mu \\
& =\frac{1}{\sqrt{l(l+1)}} \int_{\mathbb{S}^{2}}\left(\mathbf{n} \times \nabla^{*} f\right) \cdot\left(\mathbf{n} \times \nabla^{*} Y_{l, m}\right) d \mu \\
& =\frac{1}{\sqrt{l(l+1)}} \int_{\mathbb{S}^{2}} \nabla^{*} f \cdot \nabla^{*} Y_{l, m} d \mu=\frac{\left(f,-\Delta Y_{l, m}\right)}{\sqrt{l(l+1)}}
\end{aligned}
$$

where the final step follows via integration by parts. Using $-\Delta Y_{l, m}=l(l+1) Y_{l, m}$ in the last equation above then results in this:

$$
\widetilde{\mathbf{f}}(l, m)=\sqrt{l(l+1)} \widehat{f}(l, m), l \geq 1 .
$$

One can also define Sobolev spaces for tangent vector fields in a way similar to that for the scalar case. The only complication is that locally we have to deal with vector-valued functions instead of scalar-valued functions [10. Sobolev spaces of vector fields will be denoted the same way as Sobolev spaces of scalar functions, $H^{\tau}\left(\mathbb{S}^{n}\right)$; the meaning will be clear from the context. By using the Fourier coefficients in (2.2), we see that the closure of the space of divergence-free vector fields, denoted by $H_{d i v}^{\tau}\left(\mathbb{S}^{n}\right)$, comprises all divergence-free vector fields in $H^{\tau}\left(\mathbb{S}^{n}\right)$ for which

$$
\|\mathbf{f}\|_{H_{d i v}^{\tau}}^{2}:=\sum_{l=1}^{\infty} \sum_{m=1}^{2 l+1}\left(1+\lambda_{l}\right)^{\tau}|\widetilde{\mathbf{f}}(l, m)|^{2}<\infty .
$$

It is easy to show that when $\tau>1$ the functions in $H_{d i v}^{\tau}\left(\mathbb{S}^{n}\right)$ are continuous.

2.2. Positive definite kernels and native spaces. A native space is a reproducing kernel Hilbert space 13 associated with a kernel generated by a radial basis function or a spherical basis function (SBF) 42. SBFs are positive definite 
functions on the sphere with expansions of the form

$$
\psi(x, y)=\sum_{l=0}^{\infty} \widehat{\psi}(l) \underbrace{\sum_{m=1}^{2 l+1} Y_{l, m}(x) Y_{l, m}(y)}_{(2 l+1) P_{l}(x \cdot y) /(4 \pi)}, \hat{\psi}(l)>0,
$$

where $P_{l}$ is the Legendre polynomial of degree $l$. The identity $\frac{2 l+1}{4 \pi} P_{l}(x \cdot y)=$ $\sum_{m=1}^{2 l+1} Y_{l, m}(x) Y_{l, m}(y)$ is the familiar addition formula for spherical harmonics. This shows that such $\psi$ are zonal, so we use the standard abuse of notation $\psi(x, y)=$ $\psi(x \cdot y)$. Our aim here is to discuss SBFs and their associated reproducing kernel Hilbert spaces of divergence-free vector fields, and in addition discuss their relationship with native spaces of curl-free functions on $\mathbb{R}^{3}$.

2.2.1. Surface-divergence free vector fields on $\mathbb{S}^{2}$. The kernel $\Psi(x, y)$ defined in (1.2) is related to an SBF, as long as $\boldsymbol{\psi}$ is an RBF; i.e., $\boldsymbol{\psi}(x, y)=\boldsymbol{\psi}(|x-y|)$. We define the function $\psi(x, y)=\left.\psi(|x-y|)\right|_{x, y \in \mathbb{S}^{2}}$. A quick calculation gives $\psi(x, y)=$ $\psi(\sqrt{2-2(x \cdot y)})$, which shows that $\psi$ is zonal, i.e., $\psi(x, y)=\psi(x \cdot y)$. Also, by 30, Corollary 4.3], $\psi$ is an SBF. Doing a straightforward computation yields $\Psi(x, y)=$ $\mathbf{L}_{x} \mathbf{L}_{y}^{T} \psi(x, y)$, where $\mathbf{L}_{x}$ and $\mathbf{L}_{y}$ operate on the $x$ and $y$ variables, respectively.

This is a special case of the following. Let $\psi(x, y)$ be an $\operatorname{SBF}$ and define $\Psi(x, y):=$ $\mathbf{L}_{x} \mathbf{L}_{y}^{T} \psi(x, y)$. This kernel can be expanded in a series of divergence-free vector spherical harmonics,

$$
\begin{aligned}
\Psi(x, y) & =\sum_{l=1}^{\infty} \widehat{\psi}(l) \sum_{m=1}^{2 l+1} \mathbf{L}_{x} Y_{l, m}(x) \mathbf{L}_{y}^{T} Y_{l, m}(y) \\
& =\sum_{l=1}^{\infty} l(l+1) \widehat{\psi}(l) \sum_{m=1}^{2 l+1} \mathbf{y}_{l, m}(x) \mathbf{y}_{l, m}^{T}(y) .
\end{aligned}
$$

Requiring that $\widehat{\psi}(l)=\mathcal{O}\left(l^{-4-\epsilon}\right)$ is enough to ensure that the kernel is continuous in both arguments.

This kernel is strictly positive definite. Given an arbitrary set of tangent vectors $\left\{\mathbf{s}_{j}\right\}$ corresponding to the discrete set of points $X$, we have

$$
\begin{aligned}
\sum_{j, k=1}^{N} \mathbf{s}_{j}^{T} \Psi\left(x_{j}, x_{k}\right) \mathbf{s}_{k} & =\sum_{l=1}^{\infty} l(l+1) \widehat{\psi}(l) \sum_{m=1}^{2 l+1} \sum_{j, k=1}^{N} \mathbf{s}_{j}^{T} \mathbf{y}_{l, m}\left(x_{j}\right) \mathbf{y}_{l, m}^{T}\left(x_{k}\right) \mathbf{s}_{k} \\
& =\sum_{l=1}^{\infty} l(l+1) \widehat{\psi}(l) \sum_{m=1}^{2 l+1}\left|\sum_{j=1}^{N} \mathbf{s}_{j}^{T} \mathbf{y}_{l, m}\left(x_{j}\right)\right|^{2} \geq 0 .
\end{aligned}
$$

Proving that $\Psi$ is strictly positive definite requires showing that equality holding in the last line above implies that $\mathbf{s}_{k}=\mathbf{0}$ for $k=1, \ldots, N$. If equality does hold, then, because each term in the sum is nonnegative, each must vanish. Moreover, since $\hat{\psi}(l)>0$, we may divide by it to obtain $\sum_{j=1}^{N} \mathbf{s}_{j}^{T} \mathbf{y}_{l, m}\left(x_{j}\right)=0$, which holds for all $l \geq 1$ and $m=1, \ldots, 2 l+1$. Next, it is easy to show that one may choose a smooth, divergence-free vector field $\mathbf{g}$ on $\mathbb{S}^{2}$ that is supported in a small neighborhood about a fixed $x_{k}$. The neighborhood should not contain any other points of $X$, and the vector field should satisfy $\mathbf{g}\left(x_{k}\right)=\mathbf{s}_{k}$. One may then use $\mathbf{g}$ 's expansion 
in the $\mathbf{y}_{l, m}$ 's to see that

$$
\left\|\mathbf{s}_{k}\right\|^{2}=\sum_{j} \mathbf{s}_{j}^{T} \underbrace{\mathbf{g}\left(x_{j}\right)}_{\mathbf{s}_{k} \delta_{k, j}}=\sum_{l, m} \widetilde{\mathbf{g}}(l, m) \underbrace{\sum_{j} \mathbf{s}_{j}^{T} \mathbf{y}_{l, m}\left(x_{j}\right)}_{\mathbf{0}}=0,
$$

The native space associated with $\Psi(x, y)=\mathbf{L}_{x} \mathbf{L}_{y}^{T} \psi(x, y)$ is defined to be $\mathcal{N}_{\Psi}$, where

$$
\mathcal{N}_{\Psi}:=\left\{\mathbf{f} \in H_{d i v}^{0}: \sum_{l=1}^{\infty} \sum_{m=1}^{2 l+1} \frac{|\widetilde{\mathbf{f}}(l, m)|^{2}}{l(l+1) \widehat{\psi}(l)}<\infty\right\},(\mathbf{f}, \mathbf{g})_{\mathcal{N}_{\Psi}}=\sum_{l=1}^{\infty} \sum_{m=1}^{2 l+1} \frac{\widetilde{\mathbf{f}}(l, m) \widetilde{\mathbf{g}}(l, m)}{l(l+1) \widehat{\psi}(l)}
$$

It is straightforward to show that the following holds.

Theorem 2.2. Let $\psi$ be an SBF satisfying $\widehat{\psi}(l)=\mathcal{O}\left(l^{-4-\epsilon}\right)$. Then $\mathcal{N}_{\Psi}$, given above together with its inner product, is the native space (RKHS) associated with $\Psi$. Moreover, $\Psi$ is a reproducing kernel for $\mathcal{N}_{\Psi}$ in the sense that, for all $\mathbf{t}_{x} \in T_{x} \mathbb{S}^{2}$ and $\mathbf{f} \in \mathcal{N}_{\Psi}$, this holds:

$$
\left(\mathbf{f}, \Psi(\cdot, x) \mathbf{t}_{x}\right)_{\mathcal{N}_{\Psi}}=\mathbf{t}_{x}^{T} \mathbf{f}(x) .
$$

This result has several consequences. Recall that by Proposition 2.1 every $L_{2}$ surface-divergence free function satisfies $\mathbf{f}=\mathbf{L} f$ for a scalar function $f$. It is not hard to show that $\mathbf{f} \in \mathcal{N}_{\Psi}$ if and only if $f \in \mathcal{N}_{\psi}$, where $\psi$ is the SBF that generates $\Psi$. Further, by comparing the Fourier coefficients involved, if $\widehat{f}(0)=0$, then one has $\|\mathbf{f}\|_{\mathcal{N}_{\Psi}}^{2}=\|f\|_{\mathcal{N}_{\psi}}^{2}$. This is not surprising given similar results in the scalar case 42, Theorem 16.9].

Another consequence of Theorem 2.2 is that, when it is combined with (2.3), we can easily relate the native spaces to Sobolev spaces. In fact, we have a more general result relating native spaces for two SBFs. The proof of the corollary below, which is a straightforward consequence of the equality in (2.4), will be omitted.

Corollary 2.3. Let $\phi$ and $\psi$ be SBFs for which there is a constant $a>0$ such that $\widehat{\psi}(l) \leq a \widehat{\phi}(l)$ for all $l \geq 0$. Then,

$$
\left\|\sum_{j=1}^{N} \Psi\left(\cdot, x_{j}\right) \mathbf{s}_{j}\right\|_{\Psi}^{2} \leq a\left\|\sum_{j=1}^{N} \Phi\left(\cdot, x_{j}\right) \mathbf{s}_{j}\right\|_{\Phi}^{2}
$$

Moreover, if $\widehat{\psi}(l) \sim \widehat{\phi}(l)$, then $\mathcal{N}_{\Psi}=\mathcal{N}_{\Phi}$, with equivalent norms. Finally, for Sobolev spaces we have that if

$$
\widehat{\psi}(l) \sim(1+l(l+1))^{-(\tau+1)}
$$

where $\tau>1$, then $N_{\Psi}=H_{\text {div }}^{\tau}\left(\mathbb{S}^{2}\right)$, with equivalent norms.

2.2.2. Curl-free vector fields on $\mathbb{R}^{3}$ and lifting from $\mathbb{S}^{2}$ to $\mathbb{R}^{3}$. So far, we have not connected the case in which $\Psi$ comes from an SBF $\psi$ to the case in which an $\mathrm{RBF}$ $\psi$ generates $\Psi$, and hence $\Psi_{\text {curl }}$ via $(1.2)$. We want to do that now.

The native space for $\Psi_{c u r l}=-\nabla \nabla^{T} \psi$ is discussed in [9, §3.2]; it will play an important role here. Let the $\operatorname{RBF} \boldsymbol{\psi}$ be in $C^{2} \cap L_{1}$ and, in addition, suppose that $\Delta_{\mathbb{R}^{3}} \boldsymbol{\psi} \in L_{1}$. Define the space

$$
\mathcal{N}_{\Psi_{\text {curl }}}:=\left\{\mathbf{f}: \nabla \times \mathbf{f}=\mathbf{0} \text { and } \int_{\mathbb{R}^{3}} \frac{\left|\xi^{T} \widehat{\mathbf{f}}(\xi)\right|^{2}}{|\xi|^{4} \widehat{\boldsymbol{\psi}}(\xi)} d^{3} \xi<\infty\right\}
$$


and equip it with the inner product

$$
(\mathbf{f}, \mathbf{g})_{\Psi_{\text {curl }}}:=(2 \pi)^{-3 / 2} \int_{\mathbb{R}^{3}} \frac{\xi^{T} \widehat{\mathbf{f}}(\xi) \overline{\xi^{T} \widehat{\mathbf{g}}(\xi)}}{|\xi|^{4} \widehat{\boldsymbol{\psi}}(\xi)} d^{3} \xi .
$$

The space $\mathcal{N}_{\Psi_{\text {curl }}}$ is then a reproducing kernel Hilbert space, with $\Psi_{\text {curl }}(x, y)$ being the reproducing kernel in the sense that if $\mathbf{c} \in \mathbb{R}^{3}$ and $\mathbf{f} \in \mathcal{N}_{\Psi_{\text {curl }}}$, then $\mathbf{c}^{T} \mathbf{f}(x)=$ $\left(\mathbf{f}, \Psi_{\text {curl }}(\cdot, x) \mathbf{c}\right)_{\Psi_{\text {curl }}}$.

There is a relationship between the native spaces for $\Psi$ and $\Psi_{\text {curl }}$ that allows us to connect or "lift" native space results from $\mathbb{S}^{2}$ to $\mathbb{R}^{3}$. This will be very important later on. Let $x \in \mathbb{S}^{2}, \mathbf{n}_{x}$ be the outward normal to $\mathbb{S}^{2}$, and let $\mathbf{s}_{x}$ be in $T_{x} \mathbb{S}^{2}$. Then, define

$$
\grave{\mathbf{s}}_{x}:=\mathbf{n}_{x} \times \mathbf{s}_{x} .
$$

When $x, y \in \mathbb{S}^{2}$ and $\mathbf{s}_{x}$ and $\mathbf{s}_{y}$ are tangent vectors at $x$ and $y$, then from (1.2), one has that the kernels $\Psi$ and $\Psi_{\text {curl }}$ satisfy $\mathbf{s}_{x}^{T} \Psi(x, y) \mathbf{s}_{y}=\grave{\mathbf{s}}_{x}^{T} \Psi_{\text {curl }}(x, y) \grave{\mathbf{s}}_{y}$. Consequently, for an arbitrary set of tangent vectors $\left\{\mathbf{s}_{j}\right\}_{j=1}^{N}$ corresponding to the discrete set of points $X$, it follows that

$$
\sum_{j, k=1}^{N} \mathbf{s}_{j}^{T} \Psi\left(x_{j}, x_{k}\right) \mathbf{s}_{k}=\sum_{j, k=1}^{N} \grave{\mathbf{s}}_{j}^{T} \Psi_{\text {curl }}\left(x_{j}, x_{k}\right) \grave{\mathbf{s}}_{k} .
$$

Because $\Psi$ and $\Psi_{\text {curl }}$ are reproducing kernels, the previous equation also has the form

$$
\left\|\sum_{j=1}^{N} \Psi\left(\cdot, x_{j}\right) \mathbf{s}_{j}\right\|_{\Psi}^{2}=\left\|\sum_{j=1}^{N} \Psi_{\text {curl }}\left(\cdot, x_{j}\right) \grave{\mathbf{s}}_{j}\right\|_{\Psi_{\text {curl }}}^{2} .
$$

\section{Pointwise error estimates and optimal stability}

Error estimates of the interpolation process typically take place at the native space level first. In this case, the natural tool to use is the so-called power function, which we introduce shortly in the case of vector fields on the sphere. The "power function" technique is based on general approximation techniques in a Hilbert space set forth in a paper by Golumb and Weinberger [13]. There are numerous publications that use the power function to obtain error estimates for RBFs and SBFs, and we mention only a few $[18,23,45$. Our approach will be based on the concept of norming sets [18, 32.

Even though we will be working on $\mathbb{S}^{2}$, for convenience extrinsic coordinates will be used and tangent vectors will be viewed as being embedded in $\mathbb{R}^{3}$. Define the space

$$
V_{X, \Psi}:=\left\{\sum_{j=1}^{N} \Psi\left(\cdot, x_{j}\right) \mathbf{s}_{j}: \mathbf{s}_{j} \in T_{x_{j}} \mathbb{S}^{2}\right\} .
$$

For any continuous vector field $\mathbf{f}$, we let $I_{X} \mathbf{f} \in V_{X, \Psi}$ denote the interpolant of $\mathbf{f}$ on $X$. Let $\mathbf{c} \in \mathbb{R}^{3}$ be tangent to the sphere at the point $x \in \mathbb{S}^{2}$. Given $\mathbf{f} \in \mathcal{N}_{\Psi}$ and $\mathbf{g} \in V_{X, \Psi}$, one can use the reproducing kernel property of $\Psi$ together with $\mathbf{f}-I_{X} \mathbf{f}$ being perpendicular to $V_{X, \Psi}$ to get the following:

$$
\begin{aligned}
\left|\mathbf{c}^{T}\left(\mathbf{f}(x)-I_{X} \mathbf{f}(x)\right)\right| & =\left|\left(\mathbf{f}-I_{X} \mathbf{f}, \Psi(\cdot, x) \mathbf{c}\right)_{\mathcal{N}_{\Psi}}\right|=\left|\left(\mathbf{f}-I_{X} \mathbf{f}, \Psi(\cdot, x) \mathbf{c}-\mathbf{g}\right)_{\mathcal{N}_{\Psi}}\right| \\
& \leq\left\|\mathbf{f}-I_{X} \mathbf{f}\right\|_{\mathcal{N}_{\Psi}}\|\Psi(\cdot, x) \mathbf{c}-\mathbf{g}\|_{\mathcal{N}_{\Psi}} . \\
& \leq\|\mathbf{f}\|_{\mathcal{N}_{\Psi}}\|\Psi(\cdot, x) \mathbf{c}-\mathbf{g}\|_{\mathcal{N}_{\Psi}} .
\end{aligned}
$$


This leads to the estimate

$$
\left|\mathbf{c}^{T}\left(\mathbf{f}(x)-I_{X} \mathbf{f}(x)\right)\right| \leq P_{\Psi, X, \mathbf{c}}(x)\|\mathbf{f}\|_{\mathcal{N}_{\Psi}},
$$

where

$$
P_{\Psi, X, \mathbf{c}}(x):=\inf _{\mathbf{g} \in V_{X, \Psi}}\|\Psi(\cdot, x) \mathbf{c}-\mathbf{g}\|_{\mathcal{N}_{\Psi}}
$$

is called the power function. A careful analysis of the power function is central for the derivation of the pointwise error estimates.

The error estimates obtained in this section will be quite limited in scope when compared to those in the later sections. However, this exercise will not be in vain; one can also use the bounds on the power function to show that the stability estimates from the previous section are, in some sense, the best possible.

A simple Markov inequality will be useful throughout the rest of this section. Recall that at a point $x$ the surface curl-gradient is given by $\mathbf{L}=\mathbf{n} \times \nabla^{*}$. Also, every spherical harmonic of degree $l$ is given by the restriction of a homogeneous harmonic polynomial of degree $l$ on $\mathbb{R}^{3}$ to the sphere. Using these facts one can show that each Cartesian coordinate of $\mathbf{L} Y_{l, m}$ is in $\Sigma_{l}$. One may therefore use the scalar Markov inequality derived in [18, Equation 7] to obtain the following vectorial Markov inequality for $\mathbf{y} \in \boldsymbol{\Sigma}_{L}$ :

$$
|\mathbf{y}(x)-\mathbf{y}(y)|_{l_{\infty}} \leq l d(x, y)\|\mathbf{y}\|_{\infty} .
$$

3.1. Norming sets. Norming sets were first introduced in the context of RBFs and SBFs in 18. They are useful for dealing with error estimates. A more general version of them, which applies to other types of reproducing kernels, is given in 32 and will be the one employed here.

Definition 3.1. Let $V$ be finite dimensional normed linear space and let $Z \subset V^{*}$ be a finite set of $N$ functionals. We say that $Z$ is a norming set for $V$ if the mapping $T: V \rightarrow T(V) \subset R^{N}$ defined by $T(v)=(z(v))_{z \in Z}$ is injective.

The mapping $T$ is known as the sampling operator for $Z$. If $Z$ is a norming set for $V$, then $T^{-1}$ exists on the range of $T$. The norm of $T^{-1}$ is known as the norming constant. The main result on norming sets is as follows.

Proposition 3.2. Suppose $V$ is a finite-dimensional normed linear space and $Z=$ $\left\{z_{1}, z_{2}, \ldots, z_{N}\right\}$ is a norming set for $V$, and let $T$ be the corresponding sampling operator. For every $\lambda \in V^{*}$ there exists a vector $u \in \mathbb{R}^{N}$ depending only on $\lambda$ such that, for every $v \in V$,

$$
\lambda(v)=\sum_{i=1}^{N} u_{j} z_{j} \quad \text { and } \quad\|u\|_{\mathbb{R}^{N^{*}}} \leq\|\lambda\|\left\|T^{-1}\right\| .
$$

Proof. See Proposition 3.4 in 32 .

For each $x_{j} \in X$, let $\mathbf{c}_{j} \in T_{x_{j}}\left(\mathbb{S}^{2}\right)$. For our purposes, $V$ and $Z$ will be given by

$$
V=\Sigma_{L}, \quad Z=\left\{c_{j i} \delta_{x_{j}}\right\}_{x_{j} \in X, 1 \leq i \leq 3} .
$$

Next consider $Z$ as functionals on $C\left(\mathbb{S}^{2}\right)$, which motivates us to use the $l_{\infty}$ norm on $R^{N}$. Therefore, the dual norm of $\mathbb{R}^{N}$ is given by the $l_{1}$ norm.

Proposition 3.3. Suppose $X \subset \mathbb{S}^{2}$ is finite with mesh norm satisfying $h_{X} \leq 1 / 2 L$. Let $Z$ be as in (3.2). Then $Z$ is a norming set of $\boldsymbol{\Sigma}_{L}$ with norming constant bounded by 2 . 
Proof. This proof follows the proof of [18, Proposition 1], with the only difference being that here we use the vectorial Markov inequality from (3.1).

Now we apply Proposition 3.2 to get the following corollary.

Corollary 3.4. For $h_{X}$ satisfying $h_{X} \leq 1 /(2 L)$ and any continuous linear functional $\lambda$ on $\boldsymbol{\Sigma}_{L}$ with $\|\lambda\|=1$, there exists $\left\{\mathbf{c}_{j} \in T_{x_{j}}\left(\mathbb{S}^{2}\right)\right\}_{j=1}^{N}$ with $\sum_{j=1}^{N}\left\|\mathbf{c}_{j}\right\|_{1} \leq 2$ such that

$$
\lambda(\mathbf{y})=\left\langle\sum_{j=1}^{N} \mathbf{c}_{j}^{T} \delta_{x_{j}}, \mathbf{y}\right\rangle,
$$

for all $\mathbf{y} \in \boldsymbol{\Sigma}_{L}$.

In order to prove a bound on the power function, we will need a few elementary results concerning the matrix-valued function $\mathbf{L}_{x} \mathbf{L}_{y}^{T} P_{l}(x, y)$.

Lemma 3.5. Let $c \in \mathbb{R}^{3}$ and $l \in \mathbb{N}$. Then

$$
\mathbf{L}_{x} \mathbf{L}_{y}^{T} P_{l}(x, y)=\frac{4 \pi}{2 l+1} \sum_{k=1}^{2 l+1} \mathbf{L} Y_{l, k}(x) \mathbf{L}^{T} Y_{l, k}(y)
$$

and

$$
\left\|\mathbf{L}_{x} \mathbf{L}_{y}^{T} P_{l}(x, y) c\right\|_{2} \leq l(l+1)\|c\|_{2} .
$$

Proof. Using the Addition Theorem, one may represent $\mathbf{L}_{x} \mathbf{L}_{y}^{T} P_{l}(x, y)$ as

$$
\begin{aligned}
\mathbf{L}_{x} \mathbf{L}_{y}^{T} P_{l}(x, y) & =\mathbf{L}_{x} \mathbf{L}_{y}^{T}\left(\frac{4 \pi}{2 l+1} \sum_{k=1}^{2 l+1} Y_{l, k}(x) Y_{l, k}(y)\right) \\
& =\frac{4 \pi}{2 l+1} \sum_{k=1}^{2 l+1} \mathbf{L} Y_{l, k}(x) \mathbf{L}^{T} Y_{l, k}(y) .
\end{aligned}
$$

For the bound in (3.4), see [6, Equation 12.6.5].

Theorem 3.6. Let $\lambda=\mathbf{c}^{T} \delta_{x}$ be a continuous linear functional on $C\left(\mathbb{S}^{2}\right)$, where $\|\mathbf{c}\|_{1}=1$. For $h_{X}$ satisfying $h_{X} \leq 1 /(2 L)$, there exists vectors $\mathbf{c}_{j} \in T_{x_{j}} \mathbb{S}^{2}, 1 \leq j \leq$ $N$, so that for all $\mathbf{y} \in \mathbf{\Sigma}_{L}$,

$$
\left\langle\lambda-\sum_{j=1}^{N} \mathbf{c}_{j}^{T} \delta_{x_{j}}, \mathbf{y}\right\rangle=0 \quad \text { and } \quad \sum_{j=1}^{N}\left\|\mathbf{c}_{j}\right\|_{1} \leq 2 .
$$

Further, the power function $P_{\Psi, X, \mathbf{c}}(x)$ can be bounded by

$$
\left|P_{\Psi, X, \mathbf{c}}(x)\right|^{2} \leq \frac{9}{4 \pi} \sum_{l>L} \widehat{\psi}(l) l(l+1)(2 l+1) .
$$

Proof. The results in (3.5) are a direct result of Corollary 3.4. Let $\mathrm{g}$ be the Riesz representer of the continuous linear functional $-\mathbf{c}^{T} \delta_{x}+\sum_{j=1}^{N} \mathbf{c}_{j}^{T} \delta_{x_{j}}$ in $\mathcal{N}_{\Psi}$. If we define $\mathbf{c}_{0}=-\mathbf{c}$ and $x_{0}=x, \mathbf{g}$ is given by

$$
\mathbf{g}=\sum_{j=0}^{N} \Psi\left(\cdot, x_{j}\right) \mathbf{c}_{j} .
$$


Before proceeding, note that (3.5) gives us $(\mathbf{g}, \mathbf{y})_{\mathcal{N}_{\Psi}}=0$ for all $\mathbf{y} \in \boldsymbol{\Sigma}_{L}$. In particular, $\widetilde{\mathbf{g}}(l, m)=0$ for all $l \leq L$. One can bound the power function with

$$
\begin{aligned}
\left|P_{\Psi, X, \mathbf{c}}(x)\right|^{2} & \leq\|\mathbf{g}\|_{\mathcal{N}_{\Psi}}^{2}=\sum_{l=L+1}^{\infty} \sum_{m=1}^{2 l+1} \frac{\widetilde{\mathbf{g}}(l, m)^{2}}{\widehat{\psi}(l) l(l+1)} \\
& =\sum_{l=L+1}^{\infty} \widehat{\psi}(l) l(l+1) \sum_{m=1}^{2 l+1} \frac{\widetilde{\mathbf{g}}(l, m)^{2}}{(\widehat{\psi}(l) l(l+1))^{2}} .
\end{aligned}
$$

A straightforward computation of $\widetilde{\mathbf{g}}(l, m)$ yields

$$
\widetilde{\mathbf{g}}(l, m)=\widehat{\psi}(l) l(l+1) \sum_{j=0}^{N} \mathbf{c}_{j}^{T} \mathbf{y}_{l, m}\left(x_{j}\right) .
$$

Now use this and (3.3) to get

$$
\begin{aligned}
\sum_{m=1}^{2 l+1} \frac{\widetilde{\mathbf{g}}(l, m)^{2}}{(\widehat{\psi}(l) l(l+1))^{2}} & =\sum_{m=1}^{2 l+1}\left|\sum_{j=0}^{N} \mathbf{c}_{j}^{T} \mathbf{y}_{l, m}\left(x_{j}\right)\right|^{2}=\sum_{m=1}^{2 l+1} \sum_{\mu, \nu} \mathbf{c}_{\mu}^{T}\left[\mathbf{y}_{l, m}\left(x_{\mu}\right) \mathbf{y}_{l, m}^{T}\left(x_{\nu}\right)\right] \mathbf{c}_{\nu} \\
& =\frac{1}{l(l+1)} \sum_{\mu, \nu} \mathbf{c}_{\mu}^{T}\left[\sum_{m=1}^{2 l+1} \mathbf{L} Y_{l, m}\left(x_{\mu}\right) \mathbf{L}^{T} Y_{l, m}\left(x_{\nu}\right)\right] \mathbf{c}_{\nu} \\
& =\frac{2 l+1}{4 \pi l(l+1)} \sum_{\mu, \nu} \mathbf{c}_{\mu}^{T} \mathbf{L}_{x} \mathbf{L}_{y}^{T} P_{l}\left(x_{\mu}, x_{\nu}\right) \mathbf{c}_{\nu} .
\end{aligned}
$$

Next, use the fact that $l_{1}$ norms are larger than $l_{2}$ norms and the bound in (3.4) to get

$$
\begin{aligned}
\sum_{m=1}^{2 l+1} \frac{\widetilde{\mathbf{g}}(l, m)^{2}}{(\widehat{\psi}(l) l(l+1))^{2}} & \leq \frac{2 l+1}{4 \pi l(l+1)} \sum_{\mu, \nu}\left|\mathbf{c}_{\mu}^{T} \mathbf{L}_{x} \mathbf{L}_{y}^{T} P_{l}\left(x_{\mu}, x_{\nu}\right) \mathbf{c}_{\nu}\right| \\
& \leq \frac{2 l+1}{4 \pi l(l+1)} \sum_{\mu, \nu}\left\|\mathbf{c}_{\mu}\right\|_{2}\left\|\mathbf{L}_{x} \mathbf{L}_{y}^{T} P_{l}\left(x_{\mu}, x_{\nu}\right) \mathbf{c}_{\nu}\right\|_{2} \\
& \leq \frac{2 l+1}{4 \pi} \sum_{\mu, \nu}\left\|\mathbf{c}_{\mu}\right\|_{2}\left\|\mathbf{c}_{\nu}\right\|_{2}=\frac{2 l+1}{4 \pi} \sum_{\mu, \nu}\left\|\mathbf{c}_{\mu}\right\|_{1} \|_{\mathbf{c}_{\nu} \|_{1}} \\
& =\frac{2 l+1}{4 \pi}\left(\sum_{\nu=0}^{N}\left\|\mathbf{c}_{\nu}\right\|_{1}\right)^{2} .
\end{aligned}
$$

To finish the bound, use (3.5) to get $\sum_{j=0}^{N}\left\|\mathbf{c}_{j}\right\|_{1}=1+\sum_{j=1}^{N}\left\|\mathbf{c}_{j}\right\|_{1} \leq 3$. Putting all these inequalities together gives the result.

The direct consequences of this theorem are twofold. First, one can express the pointwise error of a function in the native space and its divergence-free SBF interpolant in terms of the mesh norm. Second, if the function $\psi$ satisfies $\widehat{\psi}(l) \sim$ $(1+l(l+1))^{-(\tau+1)}$ and $L$ is chosen so that $1 /(2+2 L) \leq h_{X} \leq 1 / 2 L$, then we have the estimate

$$
\left\|\mathbf{f}-I_{X} \mathbf{f}\right\|_{\infty}^{2} \leq C h_{X}^{2 \tau-2}\|\mathbf{f}\|_{\mathcal{N}_{\Psi}}^{2}
$$

Note that in this case the native space is equal to $H_{d i v}^{\tau}\left(\mathbb{S}^{2}\right)$. These are the optimal orders of $h_{X}$ in pointwise error for a function of this smoothness. 
3.2. Optimal stability. In this section we explore the stability of the interpolation matrix, denoted by $A_{X, \Psi}$, through its spectral condition number. Our methods are only valid in the case where the scalar kernel $\psi$ is the restriction to $\mathbb{S}^{2}$ of a positive definite function on $\mathbb{R}^{3}$. For practical purposes, this is a mild restriction. Indeed, as discussed in section 2.2.1, many SBFs can be obtained by restricting RBFs to spheres. For the remainder of this section, we will assume that the SBF $\psi$ is the restriction of an $\operatorname{RBF} \psi: \mathbb{R}^{3} \rightarrow \mathbb{R}$ to the sphere. In this case, stability estimates result from "lifting" the problem from the sphere back to $\mathbb{R}^{3}$.

We will relate the minimum eigenvalue of $A_{X, \Psi}$ to the minimum eigenvalue of $A_{X, \Psi_{\text {curl }}}$. Recall that $\Psi_{\text {curl }}=-\nabla \nabla^{T} \boldsymbol{\psi}$ is the $3 \times 3$ matrix kernel defined in (1.2); it can be used to generate curl-free interpolants on $\mathbb{R}^{3}$. We will make use of the following result.

Proposition 3.7 (9, Theorem 7]). Let $\boldsymbol{\psi}$ be an even, positive definite function, which possesses a positive Fourier transform $\widehat{\boldsymbol{\psi}} \in C\left(\mathbb{R}^{n} / 0\right)$. With the function

$$
M(\sigma):=\inf _{\|\xi\|_{2} \leq \sigma} \widehat{\psi}(\xi)
$$

a lower bound on $\lambda_{\min }\left(A_{X, \Psi_{\text {curl }}}\right)$ is given by

$$
\lambda_{\min }\left(A_{X, \Psi_{\text {curl }}}\right) \geq\left(\frac{\sigma^{2}}{16 \pi}\right)^{(n+2) / 2} \frac{M(\sigma) \pi}{(4 \pi)^{n} \Gamma((n+2) / 2)}
$$

for any $\sigma>0$ satisfying

$$
\sigma \geq \widetilde{C} / q_{X, \mathbb{R}^{n}}
$$

Here the constant $\widetilde{C}$ is independent of $X$ and $\boldsymbol{\psi}$.

We begin with a brief review of how to set up the $2 N \times 2 N$ interpolation matrix. Let $\mathbf{f}$ be a tangent vector field. We define the data vectors by $\mathbf{d}_{j}=\mathbf{f}\left(x_{j}\right)$ for all $x_{j} \in X$. We look for an interpolant of the form

$$
I_{X} \mathbf{f}=\sum_{k=1}^{N} \Psi\left(x, x_{k}\right) \mathbf{c}_{k},
$$

where each $\mathbf{c}_{k}$ is tangent to $\mathbb{S}^{2}$ at $x_{k}$. The interpolation conditions then become

$$
\sum_{k=1}^{N} \Psi\left(x_{j}, x_{k}\right) \mathbf{c}_{k}=\mathbf{d}_{j}, j=1, \ldots, N .
$$

It is tempting to try and solve this directly, but note that multiplication of $\mathbf{c}_{k}$ by $\Psi\left(x_{j}, x_{k}\right)$ zeros out any normal component of $\mathbf{c}_{k}$, so the system (3.6) will be singular unless we incorporate the fact that we are dealing with tangent vectors. To fix this, at each $x_{k}$ we will choose a right-handed orthonormal 3-frame. Let $\mathbf{n}_{k}$ be normal to $\mathbb{S}^{2}$ at $x_{k}$ and choose $\mathbf{e}_{k}$ to be a unit tangent vector. Now let $\mathbf{f}_{k}=\mathbf{n}_{k} \times \mathbf{e}_{k}$, which makes $\left\{\mathbf{e}_{k}, \mathbf{f}_{k}, \mathbf{n}_{k}\right\}$ our frame. With this frame one can expand the coefficient vectors in terms of an appropriate basis:

$$
\mathbf{c}_{k}=\alpha_{k} \mathbf{e}_{k}+\beta_{k} \mathbf{f}_{k} \quad \text { and } \quad \mathbf{d}_{j}=\gamma_{j} \mathbf{e}_{j}+\delta_{j} \mathbf{f}_{j} .
$$

Next, define $\widetilde{\mathbf{c}}_{k}=\left(\alpha_{k}, \beta_{k}\right)^{T}$ and $\widetilde{\mathbf{d}}_{j}=\left(\gamma_{j}, \delta_{j}\right)$. In general, if $\mathbf{t}_{k} \in T_{x_{k}} \mathbb{S}^{2}$, then $\widetilde{\mathbf{t}}_{k}$ will denote its corresponding 2-dimensional vector in terms of the basis $\left\{\mathbf{e}_{k}, \mathbf{f}_{k}, \mathbf{n}_{k}\right\}$. 
The interpolation conditions then become

$$
\sum_{k=1}^{N}\left(\begin{array}{c}
\mathbf{e}_{j}^{T} \\
\mathbf{f}_{j}^{T}
\end{array}\right) \Psi\left(x_{j}, x_{k}\right)\left(\mathbf{e}_{k} \mathbf{f}_{k}\right) \widetilde{\mathbf{c}}_{k}=\widetilde{\mathbf{d}}_{j}, j=1, \ldots, N .
$$

Now define $A_{j, k}$ to be the following $2 \times 2$ matrix

$$
A_{j, k}=\left(\begin{array}{c}
\mathbf{e}_{j}^{T} \\
\mathbf{f}_{j}^{T}
\end{array}\right) \Psi\left(x_{j}, x_{k}\right)\left(\mathbf{e}_{k} \mathbf{f}_{k}\right) .
$$

Finally, define the matrix $A_{X, \Psi}$ to be the $2 N \times 2 N$ matrix whose blocks are given by $A_{j, k}$. This is the matrix in whose stability we are interested. The stability will be determined by bounding the minimum eigenvalue from below. Since the matrix $A_{X, \Psi}$ is symmetric and positive definite, this amounts to measuring the quadratic form

$$
\widetilde{\mathbf{c}}^{T} A_{X, \Psi} \widetilde{\mathbf{c}} .
$$

The interpolation matrix is directly related to the curl-free RBF interpolation matrix in $\mathbb{R}^{3}$, denoted by $A_{X, \Psi_{\text {curl }}}$. We will show that any eigenvalue of $A_{X, \Psi}$ is also an eigenvalue of $A_{X, \Psi_{\text {curl }}}$. Once this is established, we will use Proposition 3.7 to estimate the minimal eigenvalue of $A_{X, \Psi}$.

Let $\widetilde{\mathbf{c}}$ be a unit eigenvector of $A_{X, \Psi}$ with corresponding eigenvalue $\lambda$. Let $\widetilde{\mathbf{c}}_{k}$ be the 2 vector whose components are given by the $k$ th 2 -block of $\widetilde{\mathbf{c}}$. Let $\mathbf{c}_{k}$ be the usual representation of $\widetilde{\mathbf{c}}_{k}$ in $\mathbb{R}^{3}$, and define $\mathbf{c}$ to be the $3 N \times 1$ vector whose $k$ th 3 -component block is given by $\mathbf{c}_{k}$. We have

$$
\begin{aligned}
\lambda & =\widetilde{\mathbf{c}}^{T} A_{X, \Psi} \widetilde{\mathbf{c}}=\sum_{j, k} \widetilde{\mathbf{c}}_{j}^{T} A_{j, k} \widetilde{\mathbf{c}}_{k}=\sum_{j, k} \widetilde{\mathbf{c}}_{j}^{T}\left(\begin{array}{c}
\mathbf{e}_{j}^{T} \\
\mathbf{f}_{j}^{T}
\end{array}\right) \Psi\left(x_{j}, x_{k}\right)\left(\mathbf{e}_{k} \mathbf{f}_{k}\right) \widetilde{\mathbf{c}}_{k} \\
& =\sum_{j, k} \mathbf{c}_{j}^{T} \Psi\left(x_{j}, x_{k}\right) \mathbf{c}_{k} .
\end{aligned}
$$

Rewriting $\Psi$ in terms of $\Psi_{\text {curl }}$, one continues with (3.7) to get

$$
\begin{aligned}
\sum_{j, k} \mathbf{c}_{j}^{T}\left(X_{x_{j}}^{T} \Psi_{\text {curl }}\left(x_{j}, x_{k}\right) X_{x_{k}}\right) \mathbf{c}_{k} & =\sum_{j, k}\left(\mathbf{c}_{j} \times x_{j}\right)^{T} \Psi_{\text {curl }}\left(x_{j}, x_{k}\right)\left(\mathbf{c}_{k} \times x_{k}\right) \\
& =\sum_{j, k} \grave{\mathbf{c}}_{j}^{T} \Psi_{\text {curl }}\left(x_{j}, x_{k}\right) \grave{\mathbf{c}}_{k},
\end{aligned}
$$

where $\grave{\mathbf{c}}_{k}=\mathbf{c}_{k} \times x_{k}$. This gives us the following estimate for $\lambda$,

$$
\lambda=\sum_{j, k} \grave{\mathbf{c}}_{j}^{T} \Psi_{\text {curl }}\left(x_{j}, x_{k}\right) \grave{\mathbf{c}}_{k}=\grave{\mathbf{c}}^{T} A_{X, \Psi_{\text {curl }}} \grave{\mathbf{c}} \geq \lambda_{\min }\left(A_{X, \Psi_{c u r l}}\right)\|\grave{\mathbf{c}}\|_{2}^{2},
$$

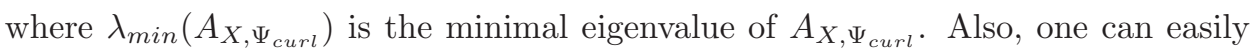
determine that $\|\grave{\mathbf{c}}\|_{2}^{2}=1$. Indeed, recall that $\mathbf{c}_{k}$ is tangent to the sphere and $x_{k}$ is a unit vector, giving us $\left\|\mathbf{c}_{k}\right\|_{2}=\left\|\grave{\mathbf{c}}_{k}\right\|_{2}$. It follows that $\|\mathbf{c}\|_{2}=\|\grave{\mathbf{c}}\|_{2}$. Also, note that $\|\widetilde{\mathbf{c}}\|_{2}=\|\mathbf{c}\|_{2}$, so $\|\mathbf{c}\|_{2}=1$. With this estimate we have the following theorem.

Theorem 3.8. Let $\boldsymbol{\psi}$ be an even positive definite function, which possesses a positive Fourier transform $\widehat{\boldsymbol{\psi}} \in C\left(\mathbb{R}^{3} / 0\right)$. Define $\psi$ by $\psi(x, y):=\left.\psi(x, y)\right|_{\mathbb{S}^{2} \times \mathbb{S}^{2}}$ and let 
$\Psi$ be the divergence-free $S B F$ on $\mathbb{S}^{2}$ generated by $\psi$. With the function

$$
M(\sigma):=\inf _{\|\xi\|_{2} \leq \sigma} \widehat{\psi}(\xi)
$$

a lower bound on $\lambda_{\min }\left(A_{X, \Psi}\right)$ is given by

$$
\lambda_{\min }\left(A_{X, \Psi}\right) \geq\left(\frac{\sigma^{2}}{16 \pi}\right)^{5 / 2} \frac{M(\sigma) \pi}{(4 \pi)^{3} \Gamma(5 / 2)}
$$

for any $\sigma>0$ satisfying

$$
\sigma \geq \widetilde{C} / q_{X, \mathbb{S}^{2}}
$$

Here the constant $\widetilde{C}$ is independent of $X$ and $\boldsymbol{\psi}$.

Proof. The result follows from Proposition 3.7 the subsequent discussion, and the fact that $q_{X, \mathbb{R}^{3}} \leq q_{X, \mathbb{S}^{2}}$.

Note that when $\psi$ satisfies

$$
\widehat{\boldsymbol{\psi}}(\xi) \sim\left(1+\|\xi\|_{2}^{2}\right)^{-\left(\tau+\frac{3}{2}\right)},
$$

then $\psi$ will satisfy (2.7), causing $\mathcal{N}_{\Psi}=H_{\text {div }}^{\tau}\left(\mathbb{S}^{2}\right)$ [30, Sect. 4.2] and [28, Sect. 4]. If we apply the theorem to this situation, we get the following corollary.

Corollary 3.9. If the Fourier transform of the RBF $\boldsymbol{\psi}$ satisfies (3.9), then the smallest eigenvalue of the interpolation matrix $A_{X, \Psi}$ can be bounded by

$$
\lambda_{\min }\left(A_{X, \Psi}\right) \geq C q_{X}^{2 \tau-2},
$$

where $C$ is a constant independent of $X$ and $\boldsymbol{\psi}$.

The following remark concerns the orders of $q_{X}$ in this estimate. In the scalar theory, when the kernel $\psi$ gives rise to a Sobolev space $H^{\tau}\left(\mathbb{R}^{n}\right)$, the resulting stability estimate is

$$
\lambda_{\min }\left(A_{X, \psi}\right) \geq C q_{X}^{2 \tau-n} .
$$

In our case, the kernel $\Psi$ generates a native space that is a subset of a Sobolev space of order $\tau$, and the dimension of the underlying space is 2 . Therefore, the orders in Corollary 3.9 should be of no surprise.

Second, one can use the bounds on the power function to show that the stability estimates given above are the best possible. To see this, one needs to derive an "uncertainty relation" similar to the one discovered by Schaback in the scalarvalued theory [39]. Given a point set $X$, a point $x_{1} \in X$ and $c_{1} \in T_{x_{1}}\left(\mathbb{S}^{2}\right)$, one can estimate the power function $P_{\Psi, X \backslash x_{1}, c_{1}}\left(x_{1}\right)$ in terms of the minimal eigenvalue of $A_{X, \Psi}$. Indeed, assuming $\left\|c_{1}\right\|_{2}=1$ and using the fact that $\Psi$ is the reproducing kernel for $\mathcal{N}_{\Psi}$ we have

$$
\begin{aligned}
{\left[P_{\Psi, X \backslash x_{1}, c_{1}}\left(x_{1}\right)\right]^{2} } & =\inf _{\mathbf{g} \in F_{X \backslash x_{1}}}\left\|\Psi\left(\cdot, x_{1}\right) c_{1}-\mathbf{g}\right\|_{\mathcal{N}_{\Psi}}^{2}=\inf _{c_{j} \in T_{x_{j}}\left(\mathbb{S}^{2}\right)} \sum_{j, k=1}^{N} c_{j}^{T} \Psi\left(x_{j}, x_{k}\right) c_{k} \\
& \geq \inf _{c_{j} \in T_{x_{j}}\left(\mathbb{S}^{2}\right)} \lambda_{\min }\left(A_{X, \Psi}\right)\left(1+\sum_{j=2}^{N}\left\|c_{j}\right\|_{2}^{2}\right)=\lambda_{\min }\left(A_{X, \Psi}\right) \\
& \geq C_{1} q_{X}^{2 \tau-2},
\end{aligned}
$$


where the last equality follows from Corollary [3.9. Also, the bounds proved in Theorem 3.6 give

$$
\left[P_{\Psi, X \backslash\left\{x_{1}\right\}, c}\left(x_{1}\right)\right]^{2} \leq C_{2} h_{X \backslash\left\{x_{1}\right\}}^{2 \tau-2} .
$$

Assuming the points in $X$ are quasi-uniform, we get

$$
C_{1} q_{X}^{2 \tau-2} \leq \lambda_{\min }\left(A_{X, \Psi}\right) \leq C_{2} q_{X}^{2 \tau-2}
$$

which shows that the stability estimates are sharp.

\section{Sobolev ERror estimates}

Until recent years one shortcoming of RBF error estimates was that they were only valid for target functions within the associated native space. However, this has been partially overcome, both on $\mathbb{R}^{n}$ and on the sphere in the scalar valued case [30, 20, 27, 34. In all of these cases, the results hold when the RBF or SBF kernel gives rise to a native space equivalent to a Sobolev space. In this section we will first discuss a variety of Sobolev error estimates for functions too rough to be in the native space, for those in the native space, and for those smoother than required for being in that space. Once we have done this, we will turn to Bernstein inequalities and corresponding inverse theorems.

4.1. Approximation with surface-divergence free vector spherical polynomials. The idea of searching for a band-limited approximating interpolant on scattered data was first introduced in 31. Not only is this an interesting result in its own right, but it turns out to be quite useful in "escaping" Sobolev native spaces, both on $\mathbb{R}^{n}$ and on the sphere [34, 27. It was shown in 8 that one can do something similar for divergence-free and curl-free functions on $\mathbb{R}^{n}$.

In these works, even for scattered data, the maximum frequency bandwidth, or Nyquist frequency, required was inversely proportional to the separation radius. For spherical harmonics in general, the index $l$ plays the role of a frequency and $L$ corresponds to a frequency bandwidth. The divergence-free "band-limited" functions on the sphere are just the vector spherical polynomials in $\boldsymbol{\Sigma}_{L}$. Now it is the goal here to prove the theorem below, which states that one can simultaneously approximate and interpolate with functions in $\boldsymbol{\Sigma}_{L}$. The proof of this theorem requires some preparation and will be postponed until that is done.

Theorem 4.1. Let $\tau>1$ and let $q=q_{X}$ for $X=\left\{x_{j}\right\}_{j=1}^{N} \subset \mathbb{S}^{2}$. For every divergence-free function $\mathbf{f} \in H_{\text {div }}^{\tau}\left(\mathbb{S}^{2}\right)$, there exists a constant $\kappa$, which depends only on $\tau$, such that if $L \geq \kappa / q$, then there is a divergence-free spherical polynomial $\mathbf{p} \in \boldsymbol{\Sigma}_{L}$ such that $\left.\mathbf{p}\right|_{X}=\left.\mathbf{f}\right|_{X}$ and $\mathbf{p}$ is a near-best approximate to $\mathbf{f}$ in the sense that

$$
\|\mathbf{f}-\mathbf{p}\|_{H_{\text {div }}^{\tau}\left(\mathbb{S}^{2}\right)} \leq\left(1+2 C_{\tau}\right) \operatorname{dist}_{H_{d i v}^{\tau}\left(\mathbb{S}^{2}\right)}\left(\mathbf{f}, \boldsymbol{\Sigma}_{L}\right),
$$

where $C_{\tau}$ is a constant depending only on $\tau$.

In 27, the authors used a "lifting" technique to relate native space norms for an $\mathrm{SBF}$ on $\mathbb{S}^{n}$ to a corresponding $\mathrm{RBF}$ on $\mathbb{R}^{n+1}$. These lifts are valid when the SBF of interest is obtained by restricting an $\mathrm{RBF}$ to $\mathbb{S}^{n}$. We wish to use a similar lifting technique here, but modified to take advantage of the relationship between surfacedivergence free kernels on $\mathbb{S}^{2}$ and curl-free kernels on $\mathbb{R}^{3}$ discussed in section 2.2.2. 
Before proceeding, we need to discuss the notation that we will use here. Let $\tau>1$ and let it be fixed throughout. Next, let $\sigma>0$ and let $L>0$ be an integer. Define the RBFs $\boldsymbol{\psi}_{\tau}$ and $\boldsymbol{\psi}_{\tau, \sigma}$ via

$$
\boldsymbol{\psi}_{\tau}(x):=\int_{\mathbb{R}^{3}} \frac{e^{i x \cdot \xi} d^{3} \xi}{(2 \pi)^{3 / 2}\left(1+|\xi|^{2}\right)^{\tau+\frac{3}{2}}} \text { and } \boldsymbol{\psi}_{\tau, \sigma}(x):=\int_{|\xi| \leq \sigma} \frac{e^{i x \cdot \xi} d^{3} \xi}{(2 \pi)^{3 / 2}\left(1+|\xi|^{2}\right)^{\tau+\frac{3}{2}}} .
$$

Let $\psi_{\tau}, \Psi_{\tau}, \Psi_{c u r l, \tau}$ be the SBF, divergence-free SBF, and curl-free RBF corresponding to $\boldsymbol{\psi}_{\tau}$, and similarly those corresponding to $\boldsymbol{\psi}_{\tau, \sigma}$. In addition, take $\psi_{\tau, L}(x \cdot y)=\sum_{l=0}^{L} \widehat{\psi}_{\tau}(l) \frac{2 l+1}{4 \pi} P_{l}(x \cdot y)$. Do the same for the other kernels. Note that both $\Psi_{\tau, L}$ and $\Psi_{\tau, \sigma, L}$ are vector spherical polynomials in $\boldsymbol{\Sigma}_{L}$. There are several facts concerning these kernels that we will employ in the proof of the theorem.

Lemma 4.2. With the RBFs in (4.1) and $\tau>1$, we have that $N_{\Psi_{\tau}}=H_{\text {div }}^{\tau}\left(\mathbb{S}^{2}\right)$, with equivalent norms. In addition, there is a constant $L_{0}>0$ such that if $L \geq L_{0}$ and $\sigma \leq e^{-1} L$, then, with $\grave{\mathbf{s}}_{j}=\mathbf{n}_{j} \times \mathbf{s}_{j}$, we have

$\left\|\sum_{j}\left(\Psi_{\tau}\left(\cdot, x_{j}\right)-\Psi_{\tau, L}\left(\cdot, x_{j}\right)\right) \mathbf{s}_{j}\right\|_{\Psi_{\tau}}^{2} \leq 2\left\|\sum_{j}\left(\Psi_{c u r l, \tau}\left(\cdot, x_{j}\right)-\Psi_{c u r l, \tau, \sigma}\left(\cdot, x_{j}\right)\right) \grave{\mathbf{s}}_{j}\right\|_{\Psi_{c u r l, \tau}}^{2}$.

Proof. By [28, Proposition 4.2], we have that $\widehat{\psi}_{\tau}(l) \sim(1+l(l+1))^{-\tau-1}$. Thus Corollary 2.3 implies that $N_{\Psi_{\tau}}=H_{\text {div }}^{\tau}\left(\mathbb{S}^{2}\right)$, with equivalent norms. Next, from the definitions of $\mathcal{N}_{\Psi_{\tau}}$ and $\Psi_{\tau, L}$, one can compute the norm on the left above to get

$$
\left\|\sum_{j}\left(\Psi_{\tau}\left(\cdot, x_{j}\right)-\Psi_{\tau, L}\left(\cdot, x_{j}\right)\right) \mathbf{s}_{j}\right\|_{\Psi_{\tau}}^{2}=\sum_{l=L+1}^{\infty} l(l+1) \widehat{\psi}_{\tau}(l) \sum_{m=1}^{2 l+1}\left|\sum_{j=1}^{N} \mathbf{s}_{j}^{T} \mathbf{y}_{l, m}\left(x_{j}\right)\right|^{2} .
$$

By [27. Equation 4.13] and the discussion preceding it, there is an $L_{0}>0$ such that for all $L \geq L_{0}$ we have that $\widehat{\psi}_{\tau, \sigma}(l) \leq \frac{1}{2} \widehat{\psi}_{\tau}(l)$, or equivalently,

$$
\widehat{\psi}_{\tau}(l) \leq 2\left(\widehat{\psi}_{\tau}(l)-\widehat{\psi}_{\tau, \sigma}(l)\right),
$$

holds whenever $l \geq L$ and $\sigma \leq e^{-1} L$. Using this, we continue with (4.2) to get

$$
\begin{aligned}
& \leq 2 \sum_{l=L+1}^{\infty} l(l+1)\left(\widehat{\psi}_{\tau}(l)-\widehat{\psi}_{\tau, \sigma}(l)\right) \sum_{m=1}^{2 l+1}\left|\sum_{j=1}^{N} \mathbf{s}_{j}^{T} \mathbf{y}_{l, m}\left(x_{j}\right)\right|^{2} \\
& <2 \sum_{l=1}^{\infty} l(l+1)\left(\widehat{\psi}_{\tau}(l)-\widehat{\psi}_{\tau, \sigma}(l)\right) \sum_{m=1}^{2 l+1}\left|\sum_{j=1}^{N} \mathbf{s}_{j}^{T} \mathbf{y}_{l, m}\left(x_{j}\right)\right|^{2} \\
& =2 \sum_{j, k} \mathbf{s}_{j}^{T}\left(\Psi_{\tau}-\Psi_{\tau, \sigma}\right)\left(x_{j}, x_{k}\right) \mathbf{s}_{k} \\
& =2 \sum_{j, k} \grave{\mathbf{s}}_{j}^{T}\left(\Psi_{c u r l, \tau}-\Psi_{c u r l, \tau, \sigma}\right)\left(x_{j}, x_{k}\right) \grave{\mathbf{s}}_{k},
\end{aligned}
$$

where the last line above follows from (2.8). Also, a direct calculation with Fourier transforms shows that the right side in the last line equals $\| \sum_{j}\left(\Psi_{\text {curl }, \tau}\left(\cdot, x_{j}\right)-\right.$ $\left.\Psi_{\text {curl }, \tau, \sigma}\left(\cdot, x_{j}\right)\right) \grave{\mathbf{s}}_{j} \|_{\Psi_{c u r l, \tau}}^{2}$. Using this in conjunction with the chain of equations above completes the proof.

We will use the following proposition, whose proof can be found in [31]. 
Proposition 4.3. Let $\mathcal{Y}$ be a (possibly complex) Banach Space, $\mathcal{V}$ a subspace of $\mathcal{Y}$, and $Z^{*}$ a finite dimensional subspace of $\mathcal{Y}^{*}$, the dual of $\mathcal{Y}$. If for every $z^{*} \in Z^{*}$ and some $\beta>1, \beta$ independent of $z^{*}$,

$$
\left\|z^{*}\right\|_{\mathcal{Y}^{*}} \leq \beta\left\|\left.z^{*}\right|_{\mathcal{V}}\right\|_{\mathcal{V}^{*}}
$$

then for $y \in \mathcal{Y}$ there exists $v \in \mathcal{V}$ such that $v$ interpolates $y$ on $Z^{*}$; that is, $z^{*}(y)=$ $z^{*}(v)$ for all $z^{*} \in Z^{*}$. In addition, $v$ approximates $y$ in the sense that $\|y-v\|_{\mathcal{Y}} \leq$ $(1+2 \beta) \operatorname{dist}(y, \mathcal{V})$.

This result will be applied to the following setup

$$
\mathcal{Y}=H_{d i v}^{\tau}\left(\mathbb{S}^{2}\right), \quad \mathcal{V}=\Sigma_{L}, \quad Z^{*}=\operatorname{span}\left\{\mathbf{s}_{j}^{T} \delta_{x_{j}}: x_{j} \in X, \mathbf{s}_{j} \in T_{x_{j}} \mathbb{S}^{2}\right\}
$$

Proof of Theorem 4.1. The proof follows along the lines of that for 27, Theorem 5.1], with modifications, of course. First note that, by Lemma 4.2 the Sobolev space $H_{\text {div }}^{\tau}\left(\mathbb{S}^{2}\right)=\mathcal{N}_{\Psi_{\tau}}$, with equivalent norms. Therefore, we have

$$
\left\|\sum_{j} \mathbf{s}_{j}^{T} \delta_{x_{j}}\right\|_{H_{d i v}^{\tau}\left(\mathbb{S}^{2}\right)^{*}} \sim\left\|\sum_{j} \mathbf{s}_{j}^{T} \delta_{x_{j}}\right\|_{\mathcal{N}_{\Psi_{\tau}}^{*}}=\left\|\sum_{j} \Psi_{\tau}\left(\cdot, x_{j}\right) \mathbf{s}_{j}\right\|_{\Psi_{\tau}} .
$$

Similarly,

$$
\left\|\sum_{j} \mathbf{s}_{j}^{T} \delta_{x_{j}} \mid \mathbf{\Sigma}_{L}\right\|_{\mathbf{\Sigma}_{L}^{*}} \sim\left\|\sum_{j} \Psi_{\tau, L}\left(\cdot, x_{j}\right) \mathbf{s}_{j}\right\|_{\Psi_{\tau}} .
$$

To simplify the notation, let $\mathbf{g}:=\sum_{j} \Psi_{\tau}\left(\cdot, x_{j}\right) \mathbf{s}_{j}, \mathbf{g}_{L}:=\sum_{j} \Psi_{\tau, L}\left(x \cdot, x_{j}\right) \mathbf{s}_{j}, \mathbf{g}_{\text {curl }}:=$ $\sum_{j} \Psi_{c u r l, \tau}\left(\cdot, x_{j}\right) \grave{\mathbf{s}}_{j}$, and $\mathbf{g}_{c u r l, \tau, \sigma}:=\sum_{j} \Psi_{c u r l, \tau, \sigma}\left(x \cdot, x_{j}\right) \grave{\mathbf{s}}_{j}$. Our aim is to obtain an estimate on the maximum of the left ratio below,

$$
\frac{\left\|\sum_{j} \mathbf{s}_{j}^{T} \delta_{x_{j}}\right\|_{H_{d i v}^{\tau}\left(\mathbb{S}^{2}\right)^{*}}}{\left\|\sum_{j} \mathbf{s}_{j}^{T} \delta_{x_{j}} \mid \boldsymbol{\Sigma}_{L}\right\|_{\boldsymbol{\Sigma}_{L}^{*}}} \leq C_{\tau} \frac{\|\mathbf{g}\|_{\Psi_{\tau}}}{\left\|\mathbf{g}_{L}\right\|_{\Psi_{\tau}}}
$$

where $C_{\tau}$ depends on the norm equivalences between the two spaces. It is easy to show that $\mathbf{g}$ and $\mathbf{g}-\mathbf{g}_{L}$ are orthogonal in $\mathcal{N}_{\Psi_{\tau}}$, so we have $\left\|\mathbf{g}_{L}\right\|_{\Psi_{\tau}}^{2}=\|\mathbf{g}\|_{\Psi_{\tau}}^{2}-$ $\left\|\mathbf{g}-\mathbf{g}_{L}\right\|_{\Psi_{\tau}}^{2}$. Therefore, finding an estimate of the form (4.3) here is equivalent to finding one of the form

$$
\frac{\left\|\mathbf{g}-\mathbf{g}_{L}\right\|_{\Psi_{\tau}}^{2}}{\|\mathbf{g}\|_{\Psi_{\tau}}^{2}} \leq 1-\frac{1}{\gamma^{2}}
$$

where $\beta / \mathbb{C}_{\tau} \sim \gamma$. By (2.9) and Lemma 4.2, with $L$ sufficiently large and $\sigma \leq e^{-1} L$, we have that

$$
\frac{\left\|\mathbf{g}-\mathbf{g}_{L}\right\|_{\Psi_{\tau}}^{2}}{\|\mathbf{g}\|_{\Psi_{\tau}}^{2}} \leq 2 \frac{\left\|\mathbf{g}_{\text {curl }}-\mathbf{g}_{\text {curl }, \sigma}\right\|_{\Psi_{c u r l, \tau}}^{2}}{\left\|\mathbf{g}_{\text {curl }}\right\|_{\Psi_{\text {curl }, \tau}}^{2}} .
$$

It was recently shown that the space $\widetilde{H}_{\text {curl }}^{\tau+1 / 2}\left(\mathbb{R}^{3}\right)$ is a reproducing kernel Hilbert space with the matrix-valued kernel, $\Psi_{\text {curl }, \tau}$ 9, Corollary 1]. That is, $\widetilde{H}_{\text {curl }}^{\tau+1 / 2}\left(\mathbb{R}^{3}\right)=$ $\mathcal{N}_{\Psi_{c u r l, \tau}}$, with the same inner product. The ratio on the right above was estimated in proving [8, Lemma 2] (technically it was shown when the kernel is divergence free, but the curl-free case is identical). In our notation, the result obtained was

$$
\frac{\left\|\mathbf{g}_{\text {curl }}-\mathbf{g}_{\text {curl }, \sigma}\right\|_{\Psi_{\text {curl }, \tau}}^{2}}{\left\|\mathbf{g}_{\text {curl }}\right\|_{\Psi_{\text {curl }, \tau}}^{2}} \leq C_{1}\left(\sigma q_{X, \mathbb{R}^{3}}\right)^{3-2(\tau+1 / 2)},
$$


where $C_{1}$ is a constant depending only on $\tau$ and $q_{X, \mathbb{R}^{3}}$ is the Euclidean separation radius for $X$ as a subset of $\mathbb{R}^{3}$. Note that $q_{X, \mathbb{R}^{3}} \geq(2 / \pi) q_{X}$. Choosing $\sigma=e^{-1} L$ in (4.5), which is as large as possible, then yields

$$
\frac{\left\|\mathbf{g}-\mathbf{g}_{L}\right\|_{\Psi_{\tau}}^{2}}{\|\mathbf{g}\|_{\Psi_{\tau}}^{2}} \leq C_{2}\left(L q_{X}\right)^{2-2 \tau}
$$

Now choose $L=\kappa / q_{X}$, where $\kappa$ is a constant large enough so that the right-hand side above is less than $3 / 4$. Thus, we have $\gamma=2$ and we have proven that an estimate of the form (4.4) holds with $\beta=2 C_{\tau}$. Thus an application of Proposition 4.3 gives the result.

We end this section with some observations from Theorem 4.1 that will be useful later. We will use $C$ to represent an arbitrary constant that depends only on $\tau$. First, if $\mathbf{p}$ is the interpolant to $\mathbf{f} \in H_{\text {div }}^{\tau}$ from $\boldsymbol{\Sigma}_{L}$, then

$$
\|\mathbf{f}-\mathbf{p}\|_{H_{d i v}^{\tau}\left(\mathbb{S}^{2}\right)} \leq C\|\mathbf{f}\|_{H_{d i v}^{\tau}\left(\mathbb{S}^{2}\right)},
$$

Also, one can relate higher ordered norms of $\mathbf{p}$ to the norm of $\mathbf{f}$ via a Bernstein inequality. For $s \geq \tau$, the Bernstein inequality $\|\mathbf{p}\|_{H_{d i v}^{s}\left(\mathbb{S}^{2}\right)} \leq L^{\tau-s}\|\mathbf{p}\|_{H_{d i v}^{\tau}\left(\mathbb{S}^{2}\right)}$ holds. Since $L \sim q_{X}$, it follows that

$$
\|\mathbf{p}\|_{H_{d i v}^{s}\left(\mathbb{S}^{2}\right)} \leq C q_{X}^{\tau-s}\|\mathbf{p}\|_{H_{d i v}^{\tau}\left(\mathbb{S}^{2}\right)} .
$$

If we add and subtract $\mathbf{f}$ to $\mathbf{p}$ on the right-hand side, use Theorem 4.1 and the previous inequality, then applying the triangle inequality gives us

$$
\|\mathbf{p}\|_{H_{d i v}^{s}\left(\mathbb{S}^{2}\right)} \leq C q_{X}^{\tau-s}\|\mathbf{f}\|_{H_{d i v}^{\tau}\left(\mathbb{S}^{2}\right)} .
$$

4.2. Error estimates within the native space. Finding error within the native space has historically been done by bounding the power function. However, in the case when the native space is Sobolev, we can make use of a recent result relating the norm of a Sobolev function defined on a domain of $\mathbb{R}^{n}$ with many zeros to its norm in another Sobolev space. Here is a statement of that result, and its proof can be found in 33 .

Proposition 4.4. Let $k$ be a positive integer, $0 \leq s<1$, and define $\tau=k+s$. Also, let $1 \leq p<\infty, 1 \leq q \leq \infty$, and let $\mu$ be an integer satisfying $k>\mu+n / p$, or $p=1$ and $k \geq \mu+n$. Also, let $X \subset \Omega$ be a discrete set with mesh norm $h_{X, \Omega}$. Then there is a constant depending only on $\Omega$ such that if $h_{X, \Omega} \leq C_{\Omega}$ and if $u \in W_{p}^{\tau}(\Omega)$ satisfies $\left.u\right|_{X}=0$, then

$$
|u|_{W_{q}^{\mu}(\Omega)} \leq C h_{X}^{\tau-\mu-n(1 / p-1 / q)_{+}}|u|_{W_{p}^{\tau}(\Omega)},
$$

where $(x)_{+}=x$ if $x \geq 0$ and is 0 otherwise. Here the constant $C$ is independent of $h_{X, \Omega}$ and $u$.

We have stated the result in a slightly stronger form that in [33, in lieu of remarks made in 34. By using this proposition on each component of a vector field, we get a similar result for vector-valued functions. Also, by mapping to $\mathbb{R}^{n}$ via charts, we gain the ability to apply the proposition to vector fields on any compact manifold. 
Theorem 4.5. Let $\tau, \mu$, and $q$ be as in Proposition 4.4 with $n=p=2$. Let $\mathbf{f}$ be a divergence-free function on $\mathbb{S}^{2}$, and let $I_{X} \mathbf{f}$ be its divergence-free SBF interpolant on $X$. If $\mathbf{f} \in H_{\text {div }}^{\tau}\left(\mathbb{S}^{2}\right)$, then

$$
\left\|\mathbf{f}-I_{X} \mathbf{f}\right\|_{W_{q}^{\mu}\left(\mathbb{S}^{2}\right)} \leq C h_{X}^{\tau-\mu-2(1 / 2-1 / q)+}\|\mathbf{f}\|_{H^{\tau}\left(\mathbb{S}^{2}\right)} .
$$

Proof. We will use the chart definition of Sobolev spaces to map to $\mathbb{R}^{2}$ via charts, use Proposition 4.4 to estimate the error on $\mathbb{R}^{2}$, and then move back to the sphere. However, in doing this, error estimates in terms of the mesh norm of the image of subsets of $X$ in $\mathbb{R}^{2}$ will be obtained instead of the mesh norm on the sphere. Luckily, an atlas $\mathcal{A}=\left\{U_{j}, \psi_{j}\right\}_{j=1}^{M}$ can be chosen so that if $\left(U_{j}, \psi_{j}\right)$ is a chart on the sphere, then an estimate of the form

$$
h_{\psi_{j}\left(X \cap U_{j}\right), \psi_{j}\left(U_{j}\right)} \leq C h_{X}
$$

holds, where $C$ is a constant independent of $X$. For a proof, see [19, Proposition $3.2]$.

Let $\left\{\chi_{j}: \mathbb{S}^{2} \rightarrow \mathbb{R}^{2}\right\}_{j=1}^{M}$ be the partition of unity associated with $\mathcal{A}$, and let $\pi_{j}$ be the projection operator associated with $\chi_{j}$. Then the norm of the error is given by

$$
\left\|\mathbf{f}-I_{X} \mathbf{f}\right\|_{W_{q}^{\mu}\left(\mathbb{S}^{2}\right)}=\left(\sum_{j=1}^{M}\left\|\pi_{j}\left(\chi_{j}\left(\mathbf{f}-I_{X} \mathbf{f}\right)\right)\right\|_{W_{q}^{\mu}\left(\mathbb{R}^{2}\right)}^{2}\right)^{1 / 2} .
$$

We will estimate the error on each patch. Since the support of $\chi_{j}$ is contained in $U_{j}$ for all $j$, we have

$$
\left\|\pi_{j}\left(\chi_{j}\left(\mathbf{f}-I_{X} \mathbf{f}\right)\right)\right\|_{W_{q}^{\mu}\left(\mathbb{R}^{2}\right)}^{2}=\left\|\pi_{j}\left(\chi_{j}\left(\mathbf{f}-I_{X} \mathbf{f}\right)\right)\right\|_{W_{q}^{\mu}\left(\psi_{j}\left(U_{j}\right)\right)}^{2} .
$$

Note that $\pi_{j}\left(\chi_{j}\left(\mathbf{f}-I_{X} \mathbf{f}\right)\right)$ is a Sobolev function with many zeros on the set $\psi_{j}(X \cap$ $U_{j}$ ). Applying Proposition 4.4 and using (4.9) gives us

$$
\left\|\pi_{j}\left(\chi_{j}\left(\mathbf{f}-I_{X} \mathbf{f}\right)\right)\right\|_{W_{q}^{\mu}\left(\psi_{j}\left(U_{j}\right)\right)} \leq C h_{X}^{\tau-\mu-2(1 / 2-1 / q)_{+}}\left\|\pi_{j}\left(\chi_{j}\left(\mathbf{f}-I_{X} \mathbf{f}\right)\right)\right\|_{W_{2}^{\tau}\left(\psi_{j}\left(U_{j}\right)\right)},
$$

where the constant is independent of $X$ and $\mathbf{f}$. Applying this estimate to all patches and using the fact that $W_{2}^{\tau}\left(\mathbb{S}^{2}\right)$ is norm equivalent to $H^{\tau}\left(\mathbb{S}^{2}\right)$ gives us

$$
\left\|\mathbf{f}-I_{X} \mathbf{f}\right\|_{W_{q}^{\mu}\left(\mathbb{S}^{2}\right)} \leq C h_{X}^{\tau-\mu-2(1 / 2-1 / q)_{+}}\left\|\mathbf{f}-I_{X} \mathbf{f}\right\|_{H^{\tau}\left(\mathbb{S}^{2}\right)} .
$$

Now recall that for kernels that satisfy (2.7), the native space is equal to $H_{d i v}^{\tau}\left(\mathbb{S}^{2}\right)$ with equivalent norms. Now use this and the fact that the RBF interpolants have a best approximation property to get

$$
\left\|\mathbf{f}-I_{X} \mathbf{f}\right\|_{H^{\tau}\left(\mathbb{S}^{2}\right)} \leq C\left\|\mathbf{f}-I_{X} \mathbf{f}\right\|_{\mathcal{N}_{\Psi}} \leq C\|\mathbf{f}\|_{\mathcal{N}_{\Psi}} \leq C\|\mathbf{f}\|_{H^{\tau}\left(\mathbb{S}^{2}\right)} .
$$

This completes the proof.

Often in applications the target function is much smoother than functions in the native space. In this case, there is a "doubling" trick from spline theory that can be used to dramatically increase the order of $h_{X}$ in the error estimates [25, 38].

Corollary 4.6. Let $\tau$ and $\mu$ be given as in Theorem 4.5. Let $\mathbf{f} \in H_{\text {div }}^{2 \tau-\mu}\left(\mathbb{S}^{2}\right)$, where $0 \leq \mu \leq \tau$. Then

$$
\left\|\mathbf{f}-I_{X} \mathbf{f}\right\|_{H^{\mu}\left(\mathbb{S}^{2}\right)} \leq C h_{X}^{2(\tau-\mu)}\|\mathbf{f}\|_{H^{2 \tau-\mu}\left(\mathbb{S}^{2}\right)}
$$


Proof. The proof of Theorem 4.5 gives the estimate

$$
\left\|\mathbf{f}-I_{X} \mathbf{f}\right\|_{H^{\mu}\left(\mathbb{S}^{2}\right)}^{2} \leq C h_{X}^{2(\tau-\mu)}\left\|\mathbf{f}-I_{X} \mathbf{f}\right\|_{H^{\tau}\left(\mathbb{S}^{2}\right)}^{2} .
$$

Recall that $\mathcal{N}_{\Psi}=H_{\text {div }}^{\tau}\left(\mathbb{S}^{2}\right)$ with equivalent norms, and $\widehat{\psi}(l) \sim\left(1+l^{2}\right)^{-(\tau+1)}$. The fact that $\mathcal{N}_{\Psi}$ is a reproducing kernel Hilbert space with kernel $\Psi$ and a quick Cauchy-Schwarz inequality gives

$$
\begin{aligned}
\left\|\mathbf{f}-I_{X} \mathbf{f}\right\|_{H^{\tau}\left(\mathbb{S}^{2}\right)}^{2} & \leq C\left\|\mathbf{f}-I_{X} \mathbf{f}\right\|_{\mathcal{N}_{\Psi}}^{2}=C\left(\mathbf{f}-I_{X} \mathbf{f}, \mathbf{f}\right)_{\mathcal{N}_{\Psi}} \\
& =C \sum_{l=1}^{\infty} \sum_{m=1}^{2 l+1} \frac{\left(\mathbf{f - I _ { X }} \mathbf{f}\right)(l, m) \widetilde{\mathbf{f}}(l, m)}{\widehat{\psi}(l) l(l+1)} \\
& =C \sum_{l=1}^{\infty} \sum_{m=1}^{2 l+1}\left(\widetilde{\mathbf{f - I _ { X }}} \mathbf{f}\right)(l, m) l^{\mu} \frac{\left(\mathbf{f - I _ { X } \mathbf { f }}\right)(l, m) \widetilde{\mathbf{f}}(l, m)}{l^{\mu} \widehat{\psi}(l) l(l+1)} \\
& \leq C\left\|\mathbf{f}-I_{X} \mathbf{f}\right\|_{H^{\mu}\left(\mathbb{S}^{2}\right)}\|\mathbf{f}\|_{H^{2 \tau-\mu}\left(\mathbb{S}^{2}\right) .}
\end{aligned}
$$

Combining this with (4.10) yields the result.

4.3. Error estimates outside of the native space. With the band-limited results and error estimates in the previous section, we are now able to state and prove the main result.

Theorem 4.7. Let $\tau \geq \beta>1$ and let $\psi$ be an SBF satisfying (2.7). Also, let $X=\left\{x_{j}\right\}_{j=1}^{N} \subset \mathbb{S}^{2}$ be a set of distinct points with mesh norm $h_{X}$, separation radius $q_{X}$ and mesh ratio $\rho_{X}=h_{X} / q_{X}$. If $\mathbf{f} \in H_{\text {div }}^{\beta}\left(\mathbb{S}^{2}\right)$, and if $\mu$ is an integer such that $0 \leq \mu \leq\lfloor\beta\rfloor-1$, we have

$$
\left\|\mathbf{f}-I_{X} \mathbf{f}\right\|_{W_{q}^{\mu}\left(\mathbb{S}^{2}\right)} \leq C \rho_{X}^{\tau-\beta} h_{X}^{\beta-\mu-2(1 / 2-1 / q)_{+}}\|\mathbf{f}\|_{H^{\beta}\left(\mathbb{S}^{2}\right)} .
$$

Proof. Using similar arguments as in the proof of Theorem 4.5. since $\mathbf{f}-I_{X} \mathbf{f}$ is a continuous Sobolev function with many zeros, the choices of $\beta$ and $\mu$ allow us to apply Proposition 4.4 to get

$$
\left\|\mathbf{f}-I_{X} \mathbf{f}\right\|_{W_{q}^{\mu}\left(\mathbb{S}^{2}\right)} \leq C h_{X}^{\beta-\mu-2(1 / 2-1 / q)_{+}}\left\|\mathbf{f}-I_{X} \mathbf{f}\right\|_{H^{\beta}\left(\mathbb{S}^{2}\right)} .
$$

The remainder of the proof boils down to estimating $\left\|\mathbf{f}-I_{X} \mathbf{f}\right\|_{H^{\beta}\left(\mathbb{S}^{2}\right)}$. Let $\mathbf{p}$ be the polynomial interpolant to $\mathbf{f}$ from Theorem 4.1. Since $\left.\mathbf{p}\right|_{X}=\left.\mathbf{f}\right|_{X}$, then $I_{X} \mathbf{f}$ is also an interpolant to $\mathbf{p}$, giving that $I_{X} \mathbf{f}=I_{X} \mathbf{p}$. Using this and a triangle inequality, we have

$$
\left\|\mathbf{f}-I_{X} \mathbf{f}\right\|_{H^{\beta}\left(\mathbb{S}^{2}\right)} \leq\|\mathbf{f}-\mathbf{p}\|_{H^{\beta}\left(\mathbb{S}^{2}\right)}+\left\|\mathbf{p}-I_{X} \mathbf{p}\right\|_{H^{\beta}\left(\mathbb{S}^{2}\right)} .
$$

It is now our aim to estimate the two terms on the right-hand side of this inequality. The first one is easily bounded by using (4.6). For the second term, note that $\mathbf{p}$ is in every Sobolev space, and use Theorem 4.5 to obtain

$$
\left\|\mathbf{p}-I_{X} \mathbf{p}\right\|_{H^{\beta}\left(\mathbb{S}^{2}\right)} \leq C h_{X}^{\tau-\beta}\|\mathbf{p}\|_{H^{\tau}\left(\mathbb{S}^{2}\right)} .
$$

Further, we can apply the Bernstein inequality in (4.7) to get

$$
C h_{X}^{\tau-\beta}\|\mathbf{p}\|_{H^{\tau}\left(\mathbb{S}^{2}\right)} \leq C h_{X}^{\tau-\beta} q_{X}^{\beta-\tau}\|\mathbf{f}\|_{H^{\beta}\left(\mathbb{S}^{2}\right)}=C \rho_{X}^{\tau-\beta}\|\mathbf{f}\|_{H^{\beta}\left(\mathbb{S}^{2}\right)} .
$$

These facts result in the estimate

$$
\left\|\mathbf{f}-I_{X} \mathbf{f}\right\|_{H^{\beta}\left(\mathbb{S}^{2}\right)} \leq C \rho_{X}^{\tau-\beta}\|\mathbf{f}\|_{H^{\beta}\left(\mathbb{S}^{2}\right)},
$$

which finishes the proof. 
4.4. A Bernstein inequality and an inverse theorem. In this section we present two results. The first is a Bernstein-type inequality for functions in the space

$$
V_{X, \Psi}=\left\{\sum_{j=1}^{N} \Psi\left(\cdot, x_{j}\right) \mathbf{s}_{j} \mid x_{j} \in X, \mathbf{s}_{j} \in T_{x_{j}}\left(\mathbb{S}^{2}\right)\right\} .
$$

This result can then be used to establish the second theorem, which is an inverse theorem characterizing the class of functions that can be approximated by certain divergence-free SBFs.

Before we present our Bernstein inequality, we state a simple but useful byproduct of applying Hölder's inequality to the series defining our Sobolev norms. Let $s, t$ be nonnegative and satisfy $1 / s+1 / t=1$. If $\alpha$ and $\beta$ are nonnegative, then the following holds for all $\mathbf{f} \in H_{d i v}^{\tau}\left(\mathbb{S}^{2}\right)$ :

$$
\|\mathbf{f}\|_{H^{\alpha / s+\beta / t}\left(\mathbb{S}^{2}\right)} \leq\|\mathbf{f}\|_{H^{\alpha}\left(\mathbb{S}^{2}\right)}^{1 / s}\|\mathbf{f}\|_{H^{\beta}\left(\mathbb{S}^{2}\right)}^{1 / t} .
$$

Theorem 4.8. Let $V_{X, \Psi}$ be as above. For $0 \leq \mu \leq \tau$, if $\mathbf{g} \in V_{X, \Psi}$, then there is a constant $C$ that is independent of $X$ and $\mathbf{g}$ such that

$$
\|\mathbf{g}\|_{H^{\mu}\left(\mathbb{S}^{2}\right)} \leq C q_{X}^{-\mu}\|\mathbf{g}\|_{L_{2}\left(\mathbb{S}^{2}\right)} .
$$

Proof. We will need a few observations from the proof of Theorem 4.1 First, we estimated the norm of a functional by measuring the norm of its Riesz representer $\mathrm{g} \in V_{X, \Psi}$. We then projected this function onto the space $\boldsymbol{\Sigma}_{L}$ to obtain the function $\mathbf{g}_{L}$. Note that this projection is orthogonal in $H^{s}\left(\mathbb{S}^{2}\right)$ for all $s \geq \tau$, which gives the equality

$$
\|\mathbf{g}\|_{H^{s}\left(\mathbb{S}^{2}\right)}^{2}=\left\|\mathbf{g}-\mathbf{g}_{L}\right\|_{H^{s}\left(\mathbb{S}^{2}\right)}^{2}+\left\|\mathbf{g}_{L}\right\|_{H^{s}\left(\mathbb{S}^{2}\right)}^{2} .
$$

By choosing $L \sim 1 / q_{X}$, we were able to obtain equation (4.4), which translates to

$$
\left\|\mathbf{g}-\mathbf{g}_{L}\right\|_{H^{\tau}\left(\mathbb{S}^{2}\right)}^{2} \leq \frac{3}{4}\|\mathbf{g}\|_{H^{\tau}\left(\mathbb{S}^{2}\right)}^{2}
$$

The result is $\|\mathbf{g}\|_{H^{\tau}\left(\mathbb{S}^{2}\right)} \leq 2\left\|\mathbf{g}_{L}\right\|_{H^{\tau}\left(\mathbb{S}^{2}\right)}$. Since $\mathbf{g}_{L} \in \boldsymbol{\Sigma}_{L}$, we have the traditional Bernstein inequality $\left\|\mathbf{g}_{L}\right\|_{H^{\tau}\left(\mathbb{S}^{2}\right)} \leq C L^{\tau}\left\|\mathbf{g}_{L}\right\|_{L_{2}\left(\mathbb{S}^{2}\right)}$. Applying (4.12) with $s=0$ and the fact that $L \sim 1 / q_{X}$ gives

$$
\|\mathbf{g}\|_{H^{\tau}\left(\mathbb{S}^{2}\right)} \leq C q_{X}^{-\tau}\|\mathbf{g}\|_{L_{2}\left(\mathbb{S}^{2}\right)} .
$$

Now apply (4.11) to $\|\mathbf{g}\|_{H^{\mu}\left(\mathbb{S}^{2}\right)}$ with $\alpha=\tau, \beta=0, s=\tau / \mu$, and $1 / t=1-\mu / \tau$ to get

$\|\mathbf{g}\|_{H^{\mu}\left(\mathbb{S}^{2}\right)} \leq\|\mathbf{g}\|_{H^{\tau}\left(\mathbb{S}^{2}\right)}^{\mu / \tau}\|\mathbf{g}\|_{L_{2}\left(\mathbb{S}^{2}\right)}^{1-\mu / \tau} \leq\left(C q_{X}^{-\tau}\|\mathbf{g}\|_{L_{2}\left(\mathbb{S}^{2}\right)}\right)^{\mu / \tau}\|\mathbf{g}\|_{L_{2}\left(\mathbb{S}^{2}\right)}^{1-\mu / \tau}=C q_{X}^{-\mu}\|\mathbf{g}\|_{L_{2}\left(\mathbb{S}^{2}\right)}$.

This leads us to an inverse theorem, which shows that the rates of approximation in the previous section are, in some sense, the best possible. As seen in the previous section, the proper orders of approximation are only guaranteed if our nodes are more or less evenly distributed, that is, $h_{X} / q_{X}$ is bounded. This motivates the following: a family $\mathcal{F}$ comprised of sets $X$ of centers is called $\rho$-uniform if every $X \in \mathcal{F}$ satisfies $\rho_{X} \leq \rho$. 
The proof of the inverse theorem requires only two things: the existence of a $\rho$-uniform family $\mathcal{F}$ (see [27, Proposition 2.1]) and an appropriate Bernstein inequality. Our proof is identical to the one given in [27, Theorem 6.2] with obvious modifications, so we refer the reader there for details.

Theorem 4.9. Let $\tau>1$ and let $\psi$ be an SBF satisfying (2.7). In addition, let $\mathcal{F}$ be a $\rho$-uniform family. If for some continuous vector field $\mathbf{f}$ there are constants $0<\mu \leq \tau$ and $c_{\mathbf{f}}>0$ such that

$$
\left\|\mathbf{f}-I_{X} \mathbf{f}\right\|_{L_{2}\left(\mathbb{S}^{2}\right)} \leq c_{\mathbf{f}} h_{X}^{\mu}
$$

holds for all $X \in \mathcal{F}$, then, for every $0 \leq \beta<\mu, \mathbf{f} \in H_{\text {div }}^{\beta}\left(\mathbb{S}^{2}\right)$.

\section{Numerical Results}

In this section, numerical results are presented which verify the predicted results for stability (Corollary [3.9), error doubling rate (Corollary 4.6), and Sobolev error estimates (Theorem 4.7).

5.1. Divergence-free kernels. Letting $x, x_{c} \in \mathbb{S}^{2}, r=\left\|x-x_{c}\right\|_{2}$, and $\boldsymbol{\psi}$ be some positive definite $\mathrm{RBF}$ on $\mathbb{R}^{3}$, the corresponding divergence-free kernel $\Psi$ is given explicitly by

$$
\Psi\left(x, x_{c}\right)=\frac{1}{r} \psi^{\prime}(r)\left(x_{c} x^{T}-x_{c}^{T} x I\right)-\frac{1}{r}\left(\frac{1}{r} \psi^{\prime}(r)\right)^{\prime}\left(x \times x_{c}\right)\left(x \times x_{c}\right)^{T},
$$

where $I$ is the 3 -by-3 identity matrix. We use four divergence-free kernels from two different classes of positive definite RBFs for testing the stability and error estimates.

The first two kernels are both generated from the restriction to the sphere of the Matérn (or Sobolev spline) class of RBFs. This class was introduced for applications in 24, and is arguably the most important and most popular family of kernels for statistical work with RBFs [12]. The Matérn RBFs are defined as

$$
\mathrm{MA}_{\nu}: \psi(r)=\frac{2^{1-\nu}}{\Gamma(\nu)}(\varepsilon r)^{\nu} K_{\nu}(\varepsilon r)
$$

where $K_{\nu}$ corresponds to the $K$-Bessel function of order $\nu$ and $\varepsilon>0$ is the free shape parameter. In the case of $\mathbb{R}^{3}$, the Fourier transform of $\boldsymbol{\psi}$ satisfies

$$
\widehat{\boldsymbol{\psi}}(\xi)=\left(1+\|\xi\|_{2}^{2}\right)^{-\left(\nu+\frac{3}{2}\right)} .
$$

Thus, the divergence-free kernel $\Psi$ on $\mathbb{S}^{2}$ generated from $\psi$ is in the Sobolev space $H_{d i v}^{\nu}\left(\mathbb{S}^{2}\right)$. For the numerical experiments, we use the $\mathrm{MA}_{\frac{7}{2}}$ and $\mathrm{MA}_{\frac{9}{2}} \mathrm{RBFs}$; see Table 1 for the explicit form of these kernels.

The last two kernels are both generated from the restriction to the sphere of Wendland's compactly supported RBFs [11. These RBFs have also been used successfully in many applications [42. The Wendland RBFs are tailored to be compactly supported, of a specific smoothness, and positive definite in the particular dimension $n$ where the underlying approximation problem is posed. They have the general form

$$
\mathrm{WE}_{n, k}: \psi(r)= \begin{cases}p_{n, k}(r), & 0 \leq r \leq \delta \\ 0, & r>\delta\end{cases}
$$


TABLE 1. The RBFs used for generating the divergence-free kernels $\Psi$ for the numerical examples. For the Matérn RBFs, $\varepsilon>0$ is called the shape parameter while $\delta>0$ is called the support radius for the Wendland RBFs.

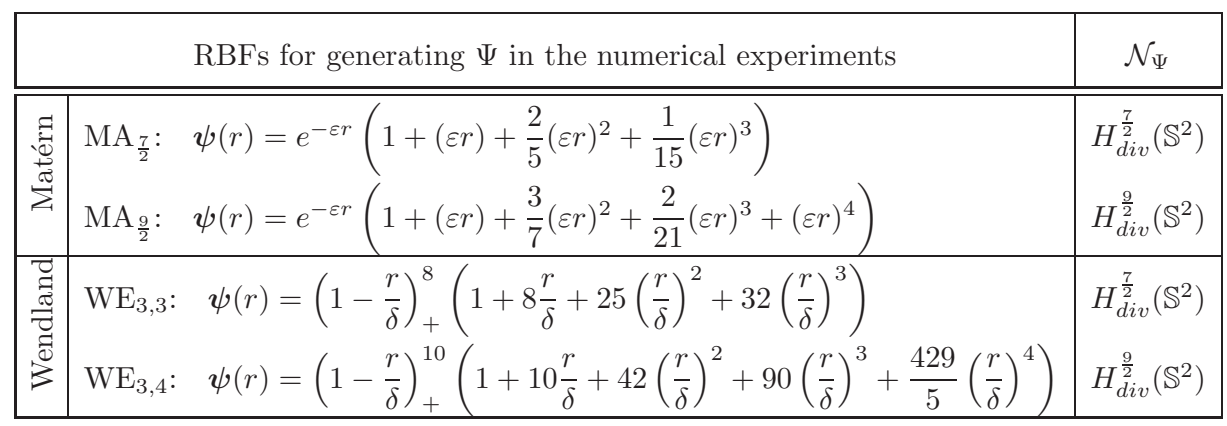

where $p_{n, k}$ is a polynomial of degree $\lfloor n / 2\rfloor+3 k+1, \psi \in C^{2 k}$, and $\delta$ is the support radius. In the case of $n=3$, the Fourier transform of $\boldsymbol{\psi}$ decays as in [42, p. 157],

$$
\widehat{\psi}(\xi) \sim\left(1+\|\xi\|_{2}^{2}\right)^{-\left(\left(k+\frac{1}{2}\right)+\frac{3}{2}\right)} .
$$

Thus, the divergence-free kernel $\Psi$ on $\mathbb{S}^{2}$ generated from $\psi$ is in the Sobolev space $H_{d i v}^{k+\frac{1}{2}}\left(\mathbb{S}^{2}\right)$. For the numerical experiments, we use the $\mathrm{WE}_{3,3}$ and $\mathrm{WE}_{3,4} \mathrm{RBFs}$; see Table 1 for the explicit form of these kernels.

As is the case with many RBFs, the Matérn and Wendland classes both feature a free parameter ( $\varepsilon$ and $\delta$, respectively). For a given node set, and corresponding data, the choice for these parameters can effect the stability and accuracy of the RBF interpolants quite dramatically. However, determining the "optimal" value for either of these parameters is neither easy nor obvious. In the case of standard RBF interpolation, several studies have been devoted to developing methods for optimally selecting these parameters (cf. 4, 36, 3, 5, in the case of the shape parameter, and [42, Ch. 15], [16, Ch. 5] in the case of the support radius). While the ideas from these papers could potentially be extended to selecting optimal shape and support radius parameters for the divergence-free interpolants, we do not pursue these extensions here. Our goal is to verify the stability and error bounds given in the previous sections. We leave the investigation of optimal parameter selection to a separate study.

In the numerical results that follow, we use $\varepsilon=5$ for $\mathrm{MA}_{\frac{7}{2}}, \varepsilon=9$ for $\mathrm{MA}_{\frac{9}{2}}$, $\delta=5 / 3$ for $\mathrm{WE}_{3,3}$, and $\delta=4 / 3$ for $\mathrm{WE}_{3,4}$. None of these values were optimized, and as shown in section 5.5 , they produce very acceptable results.

5.2. Node sets. While the divergence-free SBF interpolation method is well-posed for any distinct set of nodes $X$ on the surface of the sphere, we have chosen to use the minimum energy (ME) node sets of Womersley and Sloan 44 for our numerical examples. These node sets have several nice properties. First, both the mesh-norm $h_{X}$ and the separation radius $q_{X}$ for these node sets decay approximately uniformly like the inverse of the square root of the number of nodes $N$, i.e.,

$$
h_{X}, q_{X} \sim \frac{1}{\sqrt{N}}
$$


see Figure 1(a) This implies the mesh ratio $\rho_{X}=h_{X} / q_{X}$ appearing in the error estimate from Theorem 4.7 stays roughly constant as $N$ is increased making these nodes a $\rho$-uniform family. Second, the nodes are not oriented along any vertices or lines as illustrated in Figure 1(b) for the $N=1024 \mathrm{ME}$ node set. This emphasizes the arbitrary node layout of the divergence-free SBF technique. Third, many of these node sets are freely available for download on the web [44.

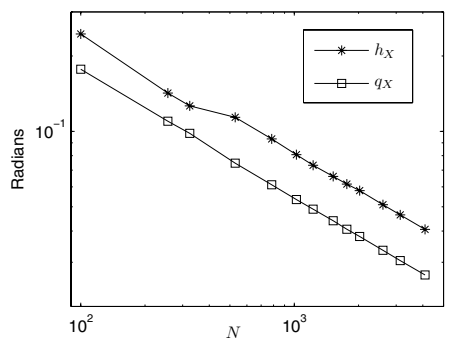

(a)

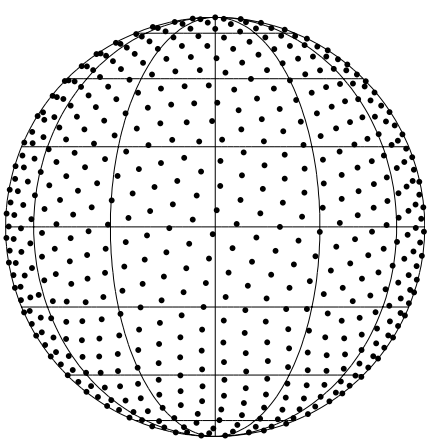

(b)

Figure 1. (a) The mesh-norm $h_{X}$ and the separation radius $q_{X}$ for minimum energy (ME) nodes sets of varying size $N$. (b) The $N=1024 \mathrm{ME}$ node set as an orthographic projection on the sphere in $\mathbb{R}^{3}$; solid black circles mark the node locations.

5.3. Test vector fields. To test the error estimates, we use three different divergence-free vector fields of varying smoothness. All of these fields are generated using "stream functions". In the descriptions of these functions that follow, we use spherical coordinates $(\theta, \lambda)$, where $\theta$ is the latitudinal direction and is measured from the equator (i.e. $-\pi / 2 \leq \theta \leq \pi / 2$ ), and $\lambda$ is the longitudinal direction $(-\pi \leq \lambda<\pi)$. With this notation, if $F$ denotes a stream function, then the divergence-free vector field $\mathbf{f}$ on the surface of the unit sphere that is generated from $F$ is given by

$$
\mathbf{f}=(\nu, \mu)^{T}=\left(\frac{1}{\cos \theta} \frac{\partial F}{\partial \lambda},-\frac{\partial F}{\partial \theta}\right),
$$

where $\nu$ and $\mu$ are the latitudinal and longitudinal components of $\mathbf{f}$, respectively.

Field 1. This field is generated from the stream function

$$
F_{1}(\theta, \lambda)=4(\cos (\alpha) \sin (\theta)-\sin (\alpha) \cos (\theta) \cos (\lambda)),
$$

and corresponds to zonal flow (or solid body rotation) at angle $\alpha$ with respect to the equator. For all experiments, we set $\alpha=\pi / 4$. This field is $C^{\infty}\left(\mathbb{S}^{2}\right)$ and will be used for testing the error estimate from Corollary 4.6. See Figure 2(a) for a plot of Field 1.

Field 2. Let

$$
g_{2}(t)=(2-2 t)^{\frac{3}{2}},
$$

and let $x$ and $x_{c}$ be points on the unit sphere with the respective spherical coordinates $(\theta, \lambda)$ and $\left(\theta_{c}, \lambda_{c}\right)$. We define $\eta$ as the dot product of $x$ and $x_{c}$, i.e.,

$$
\eta=x^{T} x_{c}=\cos (\theta) \cos \left(\lambda-\lambda_{c}\right) \cos \left(\theta_{c}\right)+\sin (\theta) \sin \left(\theta_{c}\right),
$$


and use the subscript notation $\eta_{\theta_{c}, \lambda_{c}}$ when referring to a specific point $\left(\theta_{c}, \lambda_{c}\right)$ on the unit sphere. Note that $g_{2}(\eta)$ is the standard cubic SBF centered at $x_{c}$ and has the following spherical Fourier series expansion [6]:

$$
g_{2}(\eta)=g_{2}\left(x^{T} x_{c}\right)=\sum_{l=0}^{\infty} \sum_{m=1}^{2 l+1} \widehat{g_{2}}(l) Y_{l, m}\left(x_{c}\right) Y_{l, m}(x)
$$

where

$$
\widehat{g_{2}}(l)=\frac{18 \pi}{\left(l+\frac{5}{2}\right)\left(l+\frac{3}{2}\right)\left(l+\frac{1}{2}\right)\left(l-\frac{1}{2}\right)\left(l-\frac{3}{2}\right)} .
$$

Thus, $\widehat{g_{2}}(l) \sim \ell^{-5}$ which makes $g_{2}(\eta) \in H^{\tau}\left(\mathbb{S}^{2}\right)$ for all $\tau<4$. Based on the discussion in section 2.2, any divergence-free field generated from $g_{2}(\eta)$ would be in $H_{d i v}^{\beta}\left(\mathbb{S}^{2}\right)$, for $\beta<3$.

We use $g_{2}(\eta)$ to define the stream function for the second test field as follows:

$$
F_{2}(\theta, \lambda)=g_{2}\left(\eta_{0,-\pi}\right)-g_{2}\left(\eta_{\frac{1}{10},-\frac{\pi}{2}}\right)+0.7 g_{2}\left(\eta_{-\frac{\pi}{8}, 0}\right)-g_{2}\left(\eta_{-\frac{1}{10}, \frac{\pi}{2}}\right)+0.3 g_{2}\left(\eta_{\frac{\pi}{2}-\frac{1}{10}, 0}\right) \text {; }
$$

see Figure 2(b). This field will be used to test the error estimates from Theorem 4.7.

Field 3. Let

$$
g_{3}(t)=-\frac{1}{2}\left[\left(3 t+3 \sqrt{2} a^{3 / 2}-4\right)+\left(3 t^{2}-4 t+1\right) \log (a)+(3 t-1) a \log (\sqrt{2 a}+a)\right]
$$

where $a=1-t$. This function is referred to as the "spherical spline" SBF of order 2 and has the following spherical Fourier representation [15]:

$$
g_{3}(\eta)=g_{3}\left(x^{T} x_{c}\right)=\sum_{l=0}^{\infty} \sum_{m=1}^{2 l+1} \widehat{g_{3}}(l) Y_{l, m}\left(x_{c}\right) Y_{l, m}(x) \text {, }
$$

where

$$
\widehat{g_{3}}(l)=\frac{8 \pi}{(2 l+1)(l+1)(l+2)(l+3)} .
$$

Thus, $\widehat{g_{3}}(l) \sim \ell^{-4}$ which makes $g_{3}(\eta) \in H^{\tau}\left(\mathbb{S}^{2}\right)$ for all $\tau<3$, and makes any divergence-free field generated from $g_{3}(\eta)$ in $H_{d i v}^{\beta}\left(\mathbb{S}^{2}\right)$, for $\beta<2$.

The stream function for the third test field is defined using $g_{3}(\eta)$ as follows:

$$
F_{3}(\theta, \lambda)=\int_{-\frac{\pi}{2}}^{\theta} \sin ^{14}(2 \xi) d \xi-3 g_{3}\left(\eta_{\frac{\pi}{4}, 0.25}\right) ;
$$

see Figure 2(c). We will use this field to also test the error estimates from Theorem 4.7.

All three of the test fields have a nonzero flow over both the north and south poles of the sphere, which is known to cause difficulties with many interpolation/approximation methods on spheres since, in spherical coordinates, the latitudinal and longitudinal components of the vector field will be discontinuous there [40]. The divergence-free SBF method has no such difficulties since it inherently operates on the field in Cartesian coordinates where each component of the field is continuous everywhere on the sphere including the poles. 

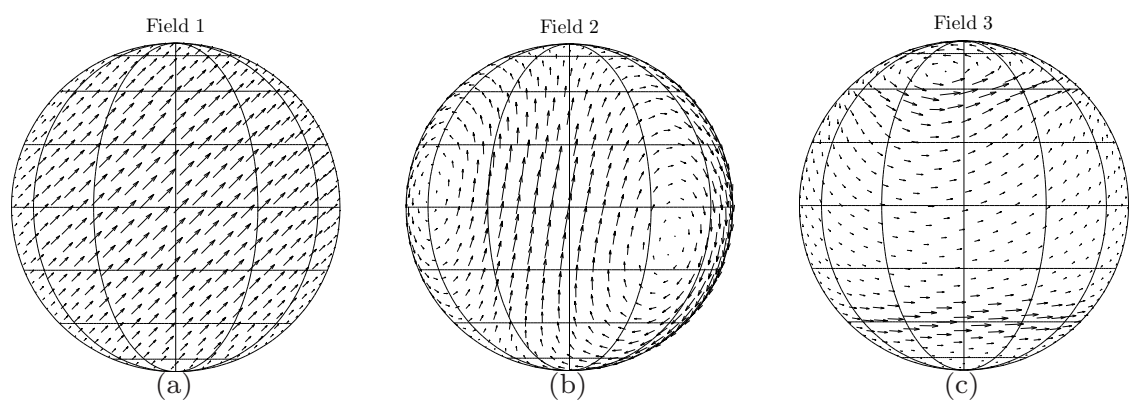

Figure 2. Vector fields used in the numerical examples. The fields are generated from the stream functions (a) $F_{1}$ in (5.1), (b) $F_{2}$ in (5.2) and (c) $F_{3}$ in (5.3). All fields are orthographic projections displayed from the following $(\theta, \lambda)$ viewpoint: (a) $(0, \pi / 2)$, (b) $(0,-\pi / 4)$, and (c) $(0,0)$.

5.4. Verification of stability estimates. Using the ME node sets, we construct the divergence-free SBF interpolation matrices $A_{X, \Psi}$ as described in section 3.2 for the RBFs listed in Table 1. For each of these matrices, we compute the minimum eigenvalue. Figure 3 displays these eigenvalues on a $\log$ - $\log$ scale as a function of the separation radius of the ME node sets. Also included in the figure is the predicted estimates for these kernels from Corollary 3.9 (see the dashed and dashdotted lines). We can see from the figure that the actual minimum eigenvalues are very well predicted by Corollary 3.9 .

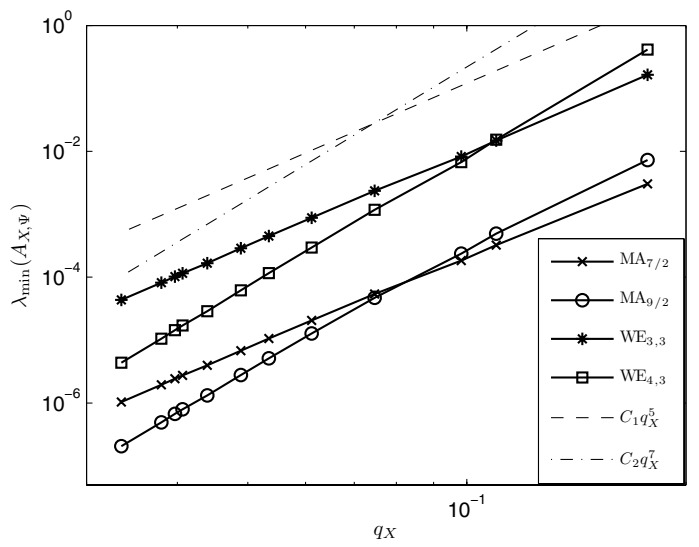

Figure 3. Minimum eigenvalue of the divergence-free SBF interpolation matrices $A_{X, \Psi}$ as a function of the separation radius $q_{X}$ of the ME node sets. The RBFs used for generating the different kernels $\Psi$ are listed in Table 1. The dashed line is the predicted estimate from Corollary 3.9 for the $\mathrm{MA}_{\frac{7}{2}}$ and $\mathrm{WE}_{3,3} \mathrm{RBFs}$, while the dash-dotted line is the prediction for the $\mathrm{MA}_{\frac{9}{2}}$ and $\mathrm{WE}_{3,4}$ RBFs. 


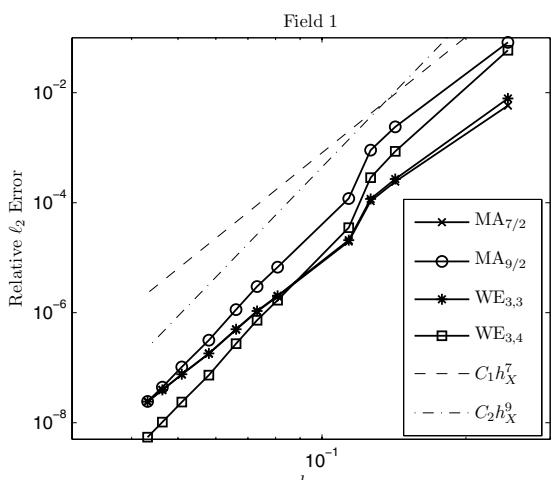

(a)

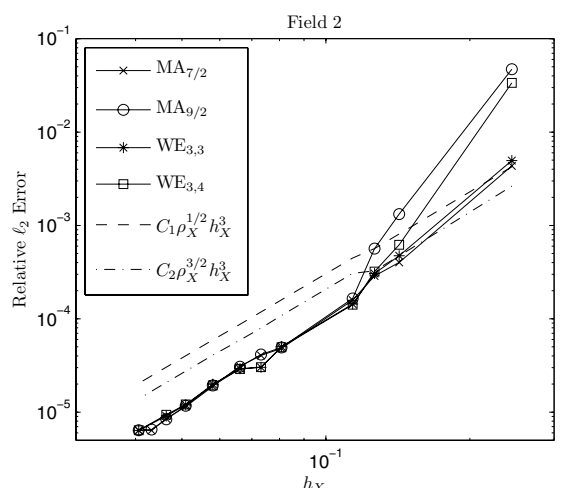

(b)

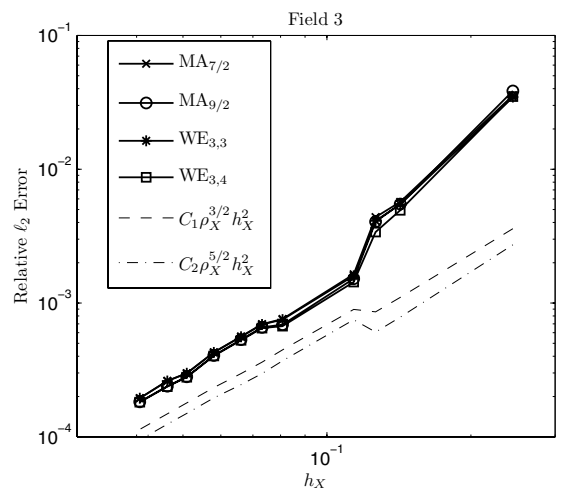

(c)

Figure 4. Relative $\ell_{2}$-errors (cf. (5.4) ) as a function of the meshnorm $h_{X}$ of the ME node sets for the divergence-free SBF interpolants to the vector fields generated from the stream functions (a) $F_{1}$ in (5.1), (b) $F_{2}$ in (5.2), and (c) $F_{3}$ in (5.3). The dashed and dash-dotted lines in each figure are defined by the plot legend and are included for comparison purposes with the results from Corollary 4.6 (figure (a)) and Theorem 4.7 (figures (b) and (c)).

5.5. Verification of error estimates. For each of the three stream functions (5.1) - (5.3) we compute the corresponding divergence-free vector field $\mathbf{f}=(\nu, \mu)$ and sample it for various ME node sets. We then compute the divergence-free SBF interpolants to these sampled fields for the RBFs listed in Table 1. These interpolants are then evaluated at 21,952 nodes which densely cover the sphere and are generated from the "spiral points" algorithm of 37. Finally, we compute the difference between the interpolants and the true vector fields at these nodes, and compute the relative $\ell_{2}$ error as follows:

$$
\text { Relative } \ell_{2} \text { error }=\frac{\left\|\underline{\mathbf{f}}-\underline{I_{X}} \mathbf{f}\right\|_{2}}{\|\underline{\mathbf{f}}\|_{2}},
$$


where $\underline{\mathbf{f}}$ and $I_{X} \mathbf{f}$ denote the respective samples of $\mathbf{f}$ and the corresponding divergence-free SBF interpolant at the evaluation nodes, and $\|\cdot\|_{2}$ is given by

$$
\|\underline{\mathbf{f}}\|_{2}=\sqrt{\sum_{j=1}^{N} \underline{\mathbf{f}}_{j}^{T} \underline{\mathbf{f}}_{j}} .
$$

The discrete $\ell_{2}$-norm can be shown to give a similar measure to the continuous Sobolev norms in Corollary 4.6 and Theorem 4.7 for the case of $\mu=0$ [33.

Figure 4(a) displays the results for Field 1. As mentioned above, this field is $C^{\infty}\left(\mathbb{S}^{2}\right)$ and thus the "doubling" error estimate from Corollary 4.6 applies. Based on this corollary, we expect the $\ell_{2}$-errors for the interpolants based on the $\mathrm{MA}_{\frac{7}{2}}$ and $\mathrm{WE}_{3,3} \mathrm{RBFs}$ to decrease like $h_{X}^{7}$, while we expect the $\ell_{2}$-errors for the interpolants based on the $\mathrm{MA}_{\frac{9}{2}}$ and $\mathrm{WE}_{3,4} \mathrm{RBFs}$ to decrease like $h_{X}^{9}$. Comparing the actual errors displayed in Figure 4(a) with the predicted ones (see the dashed and dashdotted lines), we see the estimates from Corollary 4.6 are correctly predicting the errors.

The results for Field 2 are displayed in Figure 4(b) This field is in $H_{d i v}^{\beta}\left(\mathbb{S}^{2}\right)$, $\beta<3$, and thus the error estimate from Theorem 4.7 applies. This theorem predicts that the $\ell_{2}$-errors will decrease like $\rho_{X}^{1 / 2} h_{X}^{3}$ for the divergence-free interpolants based on the $\mathrm{MA}_{\frac{7}{2}}$ and $\mathrm{WE}_{3,3}$ RBFs and will decrease like $\rho_{X}^{3 / 2} h_{X}^{3}$ for the $\mathrm{MA}_{\frac{9}{2}}$ and $\mathrm{WE}_{3,4}$ interpolants. Comparing the actual numerical results in Figure 4(b) with the predicted results (see the dashed and dash-dotted lines), we see the errors are decaying at the rates given by Theorem 4.7

The error estimates from Theorem 4.7 also apply to Field 3 since it is in $H_{d i v}^{\beta}\left(\mathbb{S}^{2}\right)$, $\beta<2$. This theorem predicts that the $\ell_{2}$-errors for the $\mathrm{MA}_{\frac{7}{2}}$ and $\mathrm{WE}_{3,3} \mathrm{RBFs}$ will decrease like $\rho_{X}^{3 / 2} h_{X}^{2}$ and for the $\mathrm{MA}_{\frac{7}{2}}$ and $\mathrm{WE}_{3,3} \mathrm{RBFs}$ like $\rho_{X}^{5 / 2} h_{X}^{2}$. The results for Field 3 are displayed in Figure 4(c) together with these predicted results (see the dashed and dash-dotted lines). We again see that the actual decay of the error is being accurately predicted by Theorem 4.7

\section{REFERENCES}

[1] Susanne C. Brenner and L. Ridgway Scott, The mathematical theory of finite element methods, second ed., Texts in Applied Mathematics, vol. 15, Springer-Verlag, New York, 2002. MR.1894376 (2003a:65103)

[2] T. C. Chen, Global water vapor flux and maintenance during FGGE, Mon. Wea. Rev. 113 (1985), 1801-1819.

[3] Gregory E. Fasshauer and Jack G. Zhang, On choosing "optimal" shape parameters for RBF approximation, Numer. Algorithms 45 (2007), no. 1-4, 345-368. MR2355993

[4] Thomas A. Foley, Near optimal parameter selection for multiquadric interpolation, J. Appl. Sci. Comput. 1 (1994), no. 1, 54-69. MR1295335

[5] Bengt Fornberg and Julia Zuev, The Runge phenomenon and spatially variable shape parameters in RBF interpolation, Comput. Math. Appl. 54 (2007), no. 3, 379-398. MR2338845 (2008e:41002)

[6] W. Freeden, T. Gervens, and M. Schreiner, Constructive approximation on the sphere, Numerical Mathematics and Scientific Computation, The Clarendon Press Oxford University Press, New York, 1998, With applications to geomathematics. MR.1694466 (2000e:41001)

[7] Willi Freeden and Theo Gervens, Vector spherical spline interpolation, Multivariate approximation theory, IV (Oberwolfach, 1989), Internat. Ser. Numer. Math., vol. 90, Birkhäuser, Basel, 1989, pp. 157-171. MR 1034306 (91a:41011)

[8] Edward J. Fuselier, Sobolev-type approximation rates for divergence-free and curl-free RBF interpolants, Math. Comp. 77 (2008), no. 263, 1407-1423. MR2398774 
[9] _ Improved stability estimates and a characterization of the native space for matrixvalued RBFs, Adv. Comp. Math. 29 (2008), no. 3, 269-290. MR2438347

[10] Peter B. Gilkey, The index theorem and the heat equation, Publish or Perish Inc., Boston, Mass., 1974, Notes by Jon Sacks, Mathematics Lecture Series, No. 4. MR0458504 (56:16704)

[11] A. E. Gill, Atmosphere-ocean dynamics, Academic Press, London, 1982.

[12] T. Gneiting, Correlation functions for atmospheric data analysis, Q. J. R. Meteorol. Soc. 125 (1999), 2449-2464.

[13] Michael Golomb and Hans F. Weinberger, Optimal approximation and error bounds, On numerical approximation. Proceedings of a Symposium, Madison, April 21-23, 1958, Edited by R. E. Langer. Publication No. 1 of the Mathematics Research Center, U.S. Army, the University of Wisconsin, The University of Wisconsin Press, Madison, Wis., 1959, pp. 117190. MR0121970 (22:12697)

[14] J. R. Holton, An introduction to dynamic meteorology, third ed., Academic Press, San Francisco, 1992.

[15] S. Hubbert, Radial basis function interpolation on the sphere, Ph.D. thesis, Imperial College, 2002.

[16] Armin Iske, Multiresolution methods in scattered data modelling, Lecture Notes in Computational Science and Engineering, vol. 37, Springer-Verlag, Berlin, 2004. MR2060191 (2005f:94023)

[17] John David Jackson, Classical electrodynamics, second ed., John Wiley \& Sons Inc., New York, 1975. MR0436782(55:9721)

[18] Kurt Jetter, Joachim Stöckler, and Joseph D. Ward, Error estimates for scattered data interpolation on spheres, Math. Comp. 68 (1999), no. 226, 733-747. MR1642746 (99i:41032)

[19] Q. T. Le Gia, F. J. Narcowich, J. D. Ward, and H. Wendland, Continuous and discrete leastsquares approximation by radial basis functions on spheres, J. Approx. Theory 143 (2006), no. 1, 124-133. MR2271729 (2007k:41078)

[20] J. Levesley and X. Sun, Approximation in rough native spaces by shifts of smooth kernels on spheres, J. Approx. Theory 133 (2005), no. 2, 269-283. MR2129483 (2005k:41024)

[21] J.-L. Lions and E. Magenes, Non-homogeneous boundary value problems and applications. Vol. I, Springer-Verlag, New York, 1972, Translated from the French by P. Kenneth, Die Grundlehren der mathematischen Wissenschaften, Band 181. MR0350177 (50:2670)

[22] C. L. Mader, Numerical modeling of water waves, CRC Press, Boca Raton, 2004.

[23] W. R. Madych and S. A. Nelson, Multivariate interpolation and conditionally positive definite functions, Approx. Theory Appl. 4 (1988), no. 4, 77-89. MR986343 (90e:41006)

[24] Bertil Matérn, Spatial variation, second ed., Lecture Notes in Statistics, vol. 36, SpringerVerlag, Berlin, 1986, With a Swedish summary. MR867886 (87m:62038)

[25] Tanya M. Morton and Marian Neamtu, Error bounds for solving pseudodifferential equations on spheres by collocation with zonal kernels, J. Approx. Theory 114 (2002), no. 2, 242-268. MR:1883408 (2002k:65200)

[26] Claus Müller, Spherical harmonics, Lecture Notes in Mathematics, vol. 17, Springer-Verlag, Berlin, 1966. MR0199449 (33:7593)

[27] Francis J. Narcowich, Xingping Sun, Joseph D. Ward, and Holger Wendland, Direct and inverse Sobolev error estimates for scattered data interpolation via spherical basis functions, Found. Comput. Math. 7 (2007), no. 3, 369-390. MR 2335250 (2008i:41023)

[28] Francis J. Narcowich, Xinping Sun, and Joseph D. Ward, Approximation power of RBFs and their associated SBFs: a connection, Adv. Comput. Math. 27 (2007), no. 1, 107-124. MR2317924

[29] Francis J. Narcowich and Joseph D. Ward, Generalized Hermite interpolation via matrixvalued conditionally positive definite functions, Math. Comp. 63 (1994), no. 208, 661-687. MR:1254147 (95c:41014)

[30] _ Scattered data interpolation on spheres: error estimates and locally supported basis functions, SIAM J. Math. Anal. 33 (2002), no. 6, 1393-1410 (electronic). MR1920637 (2003j:41021)

[31] - Scattered-data interpolation on $\mathbb{R}^{n}$ : error estimates for radial basis and bandlimited functions, SIAM J. Math. Anal. 36 (2004), no. 1, 284-300 (electronic). MR2083863 (2005g:41011) 
[32] Francis J. Narcowich, Joseph D. Ward, and Holger Wendland, Refined error estimates for radial basis function interpolation, Constr. Approx. 19 (2003), no. 4, 541-564. MR.1998904 (2004f:41019)

[33] Sobolev bounds on functions with scattered zeros, with applications to radial basis function surface fitting, Math. Comp. 74 (2005), no. 250, 743-763 (electronic). MR.2114646 (2005k:41051)

[34] Sobolev error estimates and a Bernstein inequality for scattered data interpolation via radial basis functions, Constr. Approx. 24 (2006), no. 2, 175-186. MR2239119 (2007g:41020)

[35] Francis J. Narcowich, Joseph D. Ward, and Grady B. Wright, Divergence-free RBFs on surfaces, J. Fourier Anal. Appl. 13 (2007), no. 6, 643-663. MR2350442

[36] Shmuel Rippa, An algorithm for selecting a good value for the parameter $c$ in radial basis function interpolation, Adv. Comput. Math. 11 (1999), no. 2-3, 193-210, Radial basis functions and their applications. MR 1731697

[37] E. B. Saff and A. B. J. Kuijlaars, Distributing many points on a sphere, Math. Intelligencer 19 (1997), no. 1, 5-11. MR1439152 (98h:70011)

[38] R. Schaback, Improved error bounds for scattered data interpolation by radial basis functions, Math. Comp. 68 (1999), no. 225, 201-216. MR1604379 (99d:41037)

[39] Robert Schaback, Error estimates and condition numbers for radial basis function interpolation, Adv. Comput. Math. 3 (1995), no. 3, 251-264. MR1325034 (96a:41004)

[40] Paul N. Swarztrauber, The approximation of vector functions and their derivatives on the sphere, SIAM J. Numer. Anal. 18 (1981), no. 2, 191-210. MR612138 (83e:65039)

[41] Holger Wendland, Piecewise polynomial, positive definite and compactly supported radial functions of minimal degree, Adv. Comput. Math. 4 (1995), no. 4, 389-396. MR1366510 (96h:41025)

[42] _ Scattered data approximation, Cambridge Monographs on Applied and Computational Mathematics, vol. 17, Cambridge University Press, Cambridge, 2005. MR.2131724 (2006i:41002)

[43] David L. Williamson, John B. Drake, James J. Hack, Rüdiger Jakob, and Paul N. Swarztrauber, A standard test set for numerical approximations to the shallow water equations in spherical geometry, J. Comput. Phys. 102 (1992), no. 1, 211-224. MR1177513 (93d:86006)

[44] R. S. Womersley and I. H. Sloan, Interpolation and cubature on the sphere, accessed 2008, http://web.maths.unsw.edu.au/ ${ }^{\sim}$ rsw/Sphere/

[45] Zong Min Wu and Robert Schaback, Local error estimates for radial basis function interpolation of scattered data, IMA J. Numer. Anal. 13 (1993), no. 1, 13-27. MR/1199027|(93m:65012)

Department of Mathematical Sciences, United States Military Academy, West Point, NeW YORK 10996

E-mail address: edward.fuselier@usma.edu

Department of Mathematics, Texas A\&M University, College Station, Texas 778433368

E-mail address: fnarc@math.tamu.edu

Department of Mathematics, Texas A\&M University, College Station, Texas 778433368

E-mail address: jward@math.tamu.edu

Department of Mathematics, Boise State University, Boise, Idaho 83725-1555

E-mail address: wright@diamond.boisestate.edu 